\title{
Structure and Activity Peculiarities of Quinoline and Quinoxaline Ruthenium Complexes-Novel Latent Metathesis Catalysts
}

\author{
Supplementary Information for om060091u
}

\author{
Michał Barbasiewicz,* Anna Szadkowska, Robert Bujok and Karol Grela* \\ Institute of Organic Chemistry, Polish Academy of Sciences, Kasprzaka 44/52, \\ Warsaw, 01-224, Poland \\ barabasz@icho.edu.pl \\ grela@icho.edu.pl
}

\section{Contents}

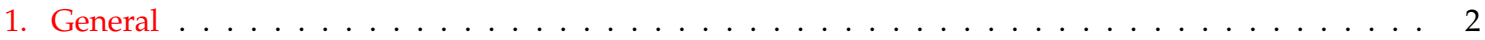

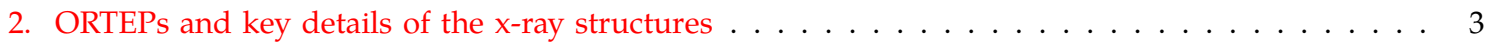

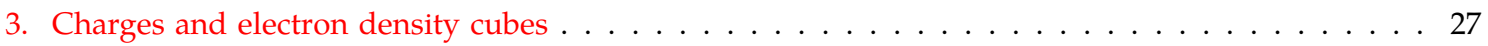

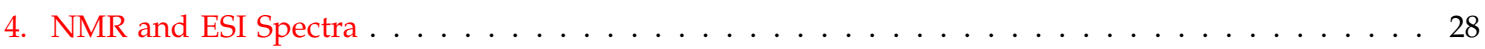




\section{General}

The catalyst preparation was carried out under Ar in pre-dried glassware using Schlenk techniques. The anhydrous solvents were dried by distillation over the following drying agents and were transferred under argon: THF (K/benzophenone), toluene $(\mathrm{Na}), n$-pentane, $n$-hexane, $\mathrm{CH}_{2} \mathrm{Cl}_{2}$ and DMF $\left(\mathrm{CaH}_{2}\right), \mathrm{Et}_{2} \mathrm{O}\left(\mathrm{LiAlH}_{4}\right), \mathrm{MeOH}(\mathrm{Mg})$. Flash column chromatography: silica gel 60 (230-400 mesh). NMR: Spectra were recorded in $\mathrm{CDCl}_{3}$; chemical shifts $(\delta)$ are given in ppm relative to TMS, coupling constants $(J)$ in Hz. IR: wavenumbers in $\mathrm{cm}^{-1}$. MS (EI, LSIMS): AMD 604 Intectra GmbH. MS (ESI): Micromass LCT with Lock-Spray unit. Injections were made after dissolving the sample in MeCN. Molecular formulas of the ruthenium compounds synthesised were confirmed by comparison of the theoretical and experimental isotope molecular patterns. GC: HP 6890 with HP 5 column. GC/MS: HP 5890 with HP 5 column. Microanalyses were provided by the Institute of Organic Chemistry, PAS, Warsaw.

8 -Vinylquinoline was prepared according to the following literature procedures. ${ }^{1} 5$-Vinylquinoxaline was prepared with analogy to the following experimental procedure. ${ }^{2}$ All commercially available chemicals were used as received.

${ }^{1}$ (a) Stille, J.K.; Echavarren, A.M. J. Am. Chem. Soc. 1987, 109, 5478. (b) Crisp, G.T.; Papadopoulos, S. Aust. J. Chem. 1989, 42, 279. (c) 8-Quinolinyl triflate is commercially available from Aldrich Inc.

2 (a) Brown, W.D.; Gouliaev, A.H. Synthesis 2002, 83. (b) van Mullekom, H. A. M.; Vekemans, J. A. J. M.; Meijer, E. W. Chem. Eur. J. 1998, 4, 1235. 


\section{ORTEPs and key details of the x-ray structures}

Figure 1. ORTEP drawing of C003501 (trans-dichloro, complex 1a. $\mathrm{C}_{6} \mathrm{H}_{6}$, cocrystallized solvent molecule has been omitted for clarity) represented by thermal ellipsoids drawn at the 50\% probability level.

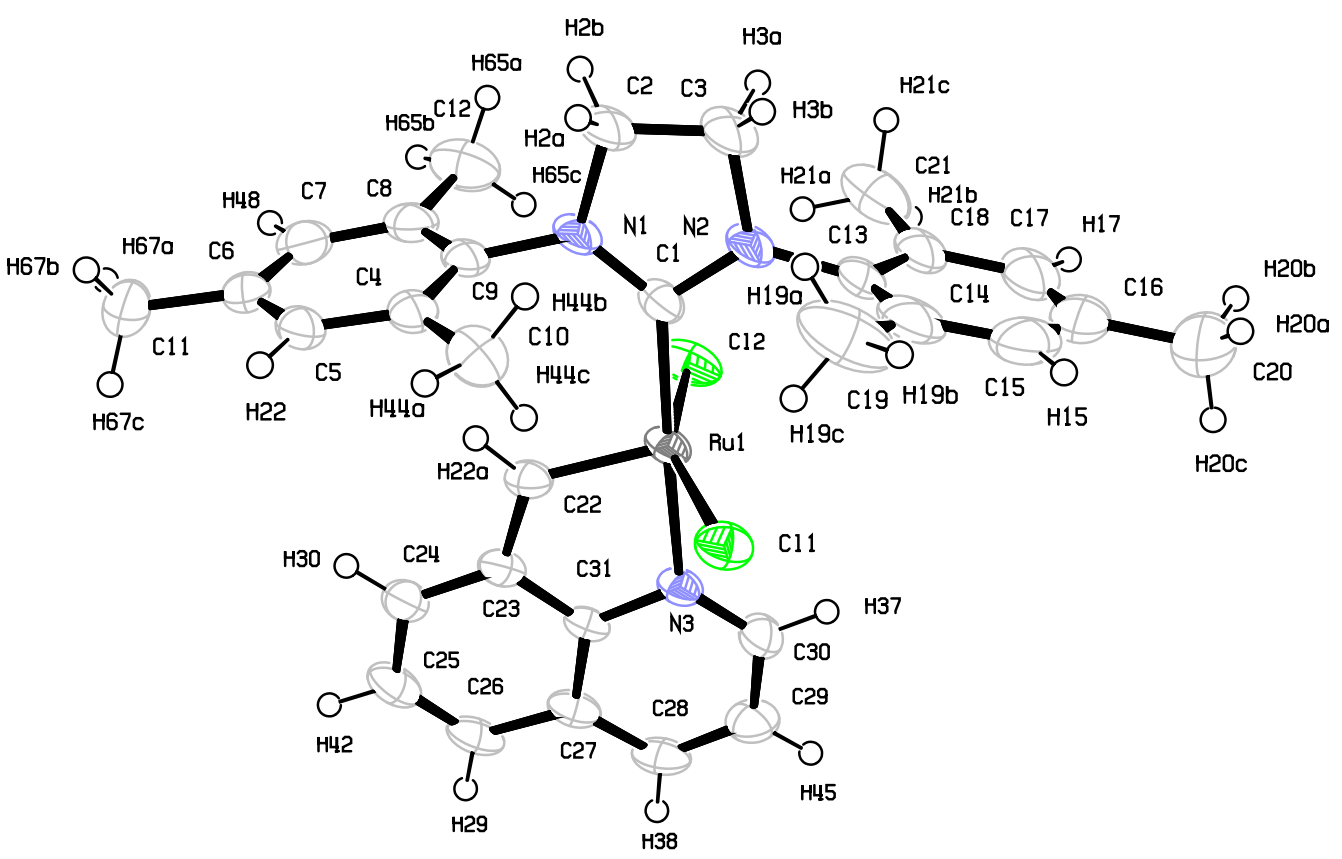

Table 1. Crystal data and structure refinement for C003501 (complex 1a).

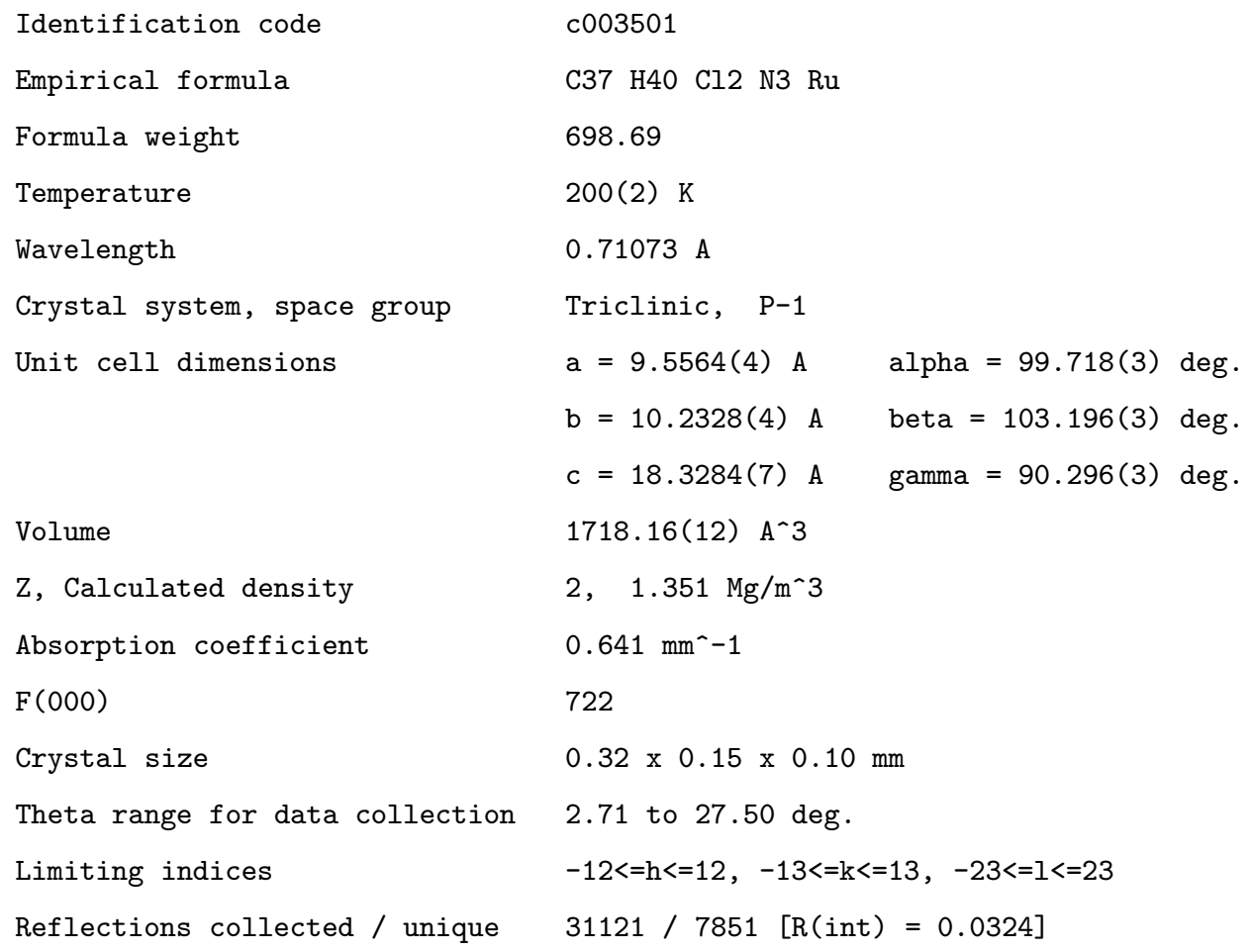


Michał Barbasiewicz et al. Structure and Activity Peculiarities of Quinoline and Quinoxaline Ruthenium Complexes. .

\begin{tabular}{|c|c|}
\hline Completeness to theta $=27.50$ & $99.3 \%$ \\
\hline Absorption correction & Numeric analytical \\
\hline Max. and min. transmission & 0.9387 and 0.8212 \\
\hline Refinement method & Full-matrix least-squares on $\mathrm{F}^{\wedge} 2$ \\
\hline Data / restraints / parameters & $7851 / 54$ / 395 \\
\hline Goodness-of-fit on $\mathrm{F}^{\wedge} 2$ & 1.084 \\
\hline Final $R$ indices $[I>2 \operatorname{sigma}(I)]$ & $\mathrm{R} 1=0.0453, \quad w R 2=0.1144$ \\
\hline $\mathrm{R}$ indices (all data) & $\mathrm{R} 1=0.0649, \quad w R 2=0.1351$ \\
\hline Extinction coefficient & $0.0217(15)$ \\
\hline Largest diff. peak and hole & 1.518 and -1.075 e. $A^{\wedge}-3$ \\
\hline
\end{tabular}

Table 2. Atomic coordinates $\left(\times 10^{4}\right)$ and equivalent isotropic displacement parameters $\left(\mathrm{A}^{2} \mathrm{x}\right.$ $10^{3}$ ) for C003501 (complex 1a). U(eq) is defined as one third of the trace of the orthogonalized $\mathrm{U}_{i j}$ tensor.

$\begin{array}{lllll} & \mathbf{x} & \mathbf{y} & \mathbf{z} & \mathbf{U}(\mathbf{e q}) \\ \mathrm{Ru}(1) & 6404(1) & 7991(1) & 2912(1) & 31(1) \\ \mathrm{Cl}(1) & 4974(1) & 9592(1) & 2358(1) & 44(1) \\ \mathrm{Cl}(2) & 8453(1) & 6738(1) & 3164(1) & 50(1) \\ \mathrm{N}(1) & 4567(4) & 5343(3) & 2283(2) & 41(1) \\ \mathrm{N}(2) & 5416(4) & 6081(3) & 1421(2) & 43(1) \\ \mathrm{N}(3) & 7663(3) & 9634(3) & 3684(2) & 32(1) \\ \mathrm{C}(1) & 5324(4) & 6374(4) & 2163(2) & 35(1) \\ \mathrm{C}(2) & 4036(5) & 4323(5) & 1593(2) & 56(1) \\ \mathrm{C}(3) & 4777(6) & 4760(5) & 1025(3) & 69(2) \\ \mathrm{C}(4) & 2721(4) & 5895(4) & 3006(2) & 37(1) \\ \mathrm{C}(5) & 2124(4) & 5713(4) & 3610(2) & 41(1) \\ \mathrm{C}(6) & 2752(5) & 4907(4) & 4129(2) & 47(1) \\ \mathrm{C}(7) & 4009(5) & 4296(4) & 4037(3) & 49(1) \\ \mathrm{C}(8) & 4636(4) & 4415(4) & 3435(2) & 43(1) \\ \mathrm{C}(9) & 3987(4) & 5237(4) & 2932(2) & 36(1) \\ \mathrm{C}(10) & 2067(5) & 6821(5) & 2467(3) & 51(1) \\ \mathrm{C}(11) & 2059(6) & 4698(5) & 4769(3) & 67(2) \\ \mathrm{C}(12) & 5960(5) & 3654(5) & 3333(3) & 66(1) \\ \mathrm{C}(13) & 6097(5) & 6834(4) & 987(2) & 44(1) \\ \mathrm{C}(14) & 5259(6) & 7667(5) & 546(3) & 63(1) \\ \mathrm{C}(15) & 5892(9) & 8285(5) & 64(3) & 78(2) \\ \mathrm{C}(16) & 7277(9) & 8056(6) & 18(3) & 84(2) \\ \mathrm{C}(17) & 8072(7) & 7198(6) & 416(3) & 73(2) \\ \mathrm{C}(18) & 7476(5) & 6529(5) & 907(2) & 51(1) \\ \mathrm{C}(19) & 3705(7) & 7841(7) & 545(3) & 94(2) \\ & & & & \end{array}$


Michał Barbasiewicz et al. Structure and Activity Peculiarities of Quinoline and Quinoxaline Ruthenium Complexes. .

$\begin{array}{lllll} & \mathbf{x} & \mathbf{y} & \mathbf{z} & \mathbf{U}(\mathbf{e q}) \\ \mathrm{C}(20) & 7923(9) & 8777(6) & -523(4) & 106(2) \\ \mathrm{C}(21) & 8322(6) & 5484(6) & 1269(3) & 71(2) \\ \mathrm{C}(22) & 5729(4) & 7901(4) & 3769(2) & 33(1) \\ \mathrm{C}(23) & 8800(3) & 4477(2) & 33(1) \\ \mathrm{C}(24) & 6393(4) & 8839(4) & 5183(2) & 38(1) \\ \mathrm{C}(25) & 6089(4) & 9768(4) & 5819(2) & 46(1) \\ \mathrm{C}(26) & 6840(5) & 10662(4) & 5746(2) & 43(1) \\ \mathrm{C}(27) & 7848(5) & 10693(4) & 5030(2) & 37(1) \\ \mathrm{C}(28) & 8173(4) & 11581(4) & 4882(2) & 44(1) \\ \mathrm{C}(29) & 9168(4) & 11481(4) & 4158(3) & 47(1) \\ \mathrm{C}(30) & 9364(5) & 10493(4) & 3570(2) & 42(1) \\ \mathrm{C}(31) & 8600(4) & 9738(3) & 4403(2) & 29(1) \\ \mathrm{C}(1 S) & 7439(4) & 2780(6) & 2347(3) & 78(2) \\ \text { C(2S) } & 9666(8) & 2054(6) & 1895(3) & 78(2) \\ \mathrm{C}(3 S) & 8447(6) & 744(6) & 1598(3) & 69(2) \\ \mathrm{C}(4 S) & 8552(6) & 140(5) & 1744(3) & 61(1) \\ \mathrm{C}(5 S) & 9834(6) & 858(6) & 2187(3) & 65(1) \\ \mathrm{C}(6 S) & 11045(6) & 2175(6) & 2489(3) & 67(2)\end{array}$

Table 3. Bond lengths $[\AA \mathrm{A}]$ and angles [deg] for C003501 (complex 1a).

$\begin{array}{ll}\mathrm{Ru}(1)-\mathrm{C}(22) & 1.846(4) \\ \mathrm{Ru}(1)-\mathrm{C}(1) & 2.063(4) \\ \mathrm{Ru}(1)-\mathrm{N}(3) & 2.162(3) \\ \mathrm{Ru}(1)-\mathrm{Cl}(2) & 2.3466(10) \\ \mathrm{Ru}(1)-\mathrm{Cl}(1) & 2.3521(10) \\ \mathrm{N}(1)-\mathrm{C}(1) & 1.352(5) \\ \mathrm{N}(1)-\mathrm{C}(9) & 1.441(5) \\ \mathrm{N}(1)-\mathrm{C}(2) & 1.484(5) \\ \mathrm{N}(2)-\mathrm{C}(1) & 1.365(5) \\ \mathrm{N}(2)-\mathrm{C}(13) & 1.444(5) \\ \mathrm{N}(2)-\mathrm{C}(3) & 1.478(5) \\ \mathrm{N}(3)-\mathrm{C}(30) & 1.326(5) \\ \mathrm{N}(3)-\mathrm{C}(31) & 1.369(5) \\ \mathrm{C}(2)-\mathrm{C}(3) & 1.509(6) \\ \mathrm{C}(2)-\mathrm{H}(2 \mathrm{~A}) & 0.9900 \\ \mathrm{C}(2)-\mathrm{H}(2 \mathrm{~B}) & 0.9900 \\ \mathrm{C}(3)-\mathrm{H}(3 \mathrm{~A}) & 0.9900 \\ \mathrm{C}(3)-\mathrm{H}(3 \mathrm{~B}) & 0.9900\end{array}$


Michał Barbasiewicz et al. Structure and Activity Peculiarities of Quinoline and Quinoxaline Ruthenium Complexes...

\begin{tabular}{|c|c|}
\hline$C(4)-C(5)$ & $1.393(5)$ \\
\hline$C(4)-C(9)$ & $1.410(5)$ \\
\hline$C(4)-C(10)$ & $1.519(6)$ \\
\hline$C(5)-C(6)$ & $1.399(6)$ \\
\hline $\mathrm{C}(5)-\mathrm{H}(22)$ & 0.9500 \\
\hline$C(6)-C(7)$ & $1.389(6)$ \\
\hline$C(6)-C(11)$ & $1.516(6)$ \\
\hline$C(7)-C(8)$ & $1.391(6)$ \\
\hline $\mathrm{C}(7)-\mathrm{H}(48)$ & 0.9500 \\
\hline$C(8)-C(9)$ & $1.396(6)$ \\
\hline$C(8)-C(12)$ & $1.521(6)$ \\
\hline $\mathrm{C}(10)-\mathrm{H}(44 \mathrm{~A})$ & 0.9800 \\
\hline $\mathrm{C}(10)-\mathrm{H}(44 \mathrm{~B})$ & 0.9800 \\
\hline $\mathrm{C}(10)-\mathrm{H}(44 \mathrm{C})$ & 0.9800 \\
\hline $\mathrm{C}(11)-\mathrm{H}(67 \mathrm{~A})$ & 0.9800 \\
\hline $\mathrm{C}(11)-\mathrm{H}(67 \mathrm{~B})$ & 0.9800 \\
\hline $\mathrm{C}(11)-\mathrm{H}(67 \mathrm{C})$ & 0.9800 \\
\hline $\mathrm{C}(12)-\mathrm{H}(65 \mathrm{~A})$ & 0.9800 \\
\hline $\mathrm{C}(12)-\mathrm{H}(65 \mathrm{~B})$ & 0.9800 \\
\hline $\mathrm{C}(12)-\mathrm{H}(65 \mathrm{C})$ & 0.9800 \\
\hline$C(13)-C(18)$ & $1.391(6)$ \\
\hline$C(13)-C(14)$ & $1.402(7)$ \\
\hline$C(14)-C(15)$ & $1.404(8)$ \\
\hline$C(14)-C(19)$ & $1.496(8)$ \\
\hline$C(15)-C(16)$ & $1.364(9)$ \\
\hline $\mathrm{C}(15)-\mathrm{H}(15)$ & 0.9500 \\
\hline$C(16)-C(17)$ & $1.360(9)$ \\
\hline$C(16)-C(20)$ & $1.559(8)$ \\
\hline$C(17)-C(18)$ & $1.428(7)$ \\
\hline $\mathrm{C}(17)-\mathrm{H}(17)$ & 0.9500 \\
\hline$C(18)-C(21)$ & $1.497(7)$ \\
\hline $\mathrm{C}(19)-\mathrm{H}(19 \mathrm{~A})$ & 0.9800 \\
\hline C(19)-H(19B) & 0.9800 \\
\hline C(19)-H(19C) & 0.9800 \\
\hline $\mathrm{C}(20)-\mathrm{H}(20 \mathrm{~A})$ & 0.9800 \\
\hline $\mathrm{C}(20)-\mathrm{H}(20 \mathrm{~B})$ & 0.9800 \\
\hline $\mathrm{C}(20)-\mathrm{H}(20 \mathrm{C})$ & 0.9800 \\
\hline $\mathrm{C}(21)-\mathrm{H}(21 \mathrm{~A})$ & 0.9800 \\
\hline $\mathrm{C}(21)-\mathrm{H}(21 \mathrm{~B})$ & 0.9800 \\
\hline $\mathrm{C}(21)-\mathrm{H}(21 \mathrm{C})$ & 0.9800 \\
\hline$C(22)-C(23)$ & $1.465(5)$ \\
\hline $\mathrm{C}(22)-\mathrm{H}(12 \mathrm{~A})$ & 0.9900 \\
\hline
\end{tabular}


Michał Barbasiewicz et al. Structure and Activity Peculiarities of Quinoline and Quinoxaline Ruthenium Complexes...

\begin{tabular}{|c|c|}
\hline $\mathrm{C}(22)-\mathrm{H}(12 \mathrm{~B})$ & 0.9900 \\
\hline$C(23)-C(24)$ & $1.383(5)$ \\
\hline$C(23)-C(31)$ & $1.427(5)$ \\
\hline$C(24)-C(25)$ & $1.419(6)$ \\
\hline $\mathrm{C}(24)-\mathrm{H}(30)$ & 0.9500 \\
\hline$C(25)-C(26)$ & $1.370(6)$ \\
\hline $\mathrm{C}(25)-\mathrm{H}(42)$ & 0.9500 \\
\hline$C(26)-C(27)$ & $1.422(6)$ \\
\hline $\mathrm{C}(26)-\mathrm{H}(29)$ & 0.9500 \\
\hline$C(27)-C(28)$ & $1.413(6)$ \\
\hline$C(27)-C(31)$ & $1.419(5)$ \\
\hline$C(28)-C(29)$ & $1.369(6)$ \\
\hline $\mathrm{C}(28)-\mathrm{H}(38)$ & 0.9500 \\
\hline$C(29)-C(30)$ & $1.408(5)$ \\
\hline $\mathrm{C}(29)-\mathrm{H}(45)$ & 0.9500 \\
\hline $\mathrm{C}(30)-\mathrm{H}(37)$ & 0.9500 \\
\hline$C(1 S)-C(6 S)$ & $1.366(8)$ \\
\hline $\mathrm{C}(1 S)-\mathrm{C}(2 \mathrm{~S})$ & $1.391(8)$ \\
\hline $\mathrm{C}(1 \mathrm{~S})-\mathrm{H}(77)$ & 0.9500 \\
\hline $\mathrm{C}(2 \mathrm{~S})-\mathrm{C}(3 \mathrm{~S})$ & $1.373(8)$ \\
\hline $\mathrm{C}(2 \mathrm{~S})-\mathrm{H}(74)$ & 0.9500 \\
\hline$C(3 S)-C(4 S)$ & $1.367(7)$ \\
\hline $\mathrm{C}(3 \mathrm{~S})-\mathrm{H}(76)$ & 0.9500 \\
\hline$C(4 S)-C(5 S)$ & $1.379(7)$ \\
\hline $\mathrm{C}(4 \mathrm{~S})-\mathrm{H}(75)$ & 0.9500 \\
\hline$C(5 S)-C(6 S)$ & $1.380(7)$ \\
\hline $\mathrm{C}(5 \mathrm{~S})-\mathrm{H}(72)$ & 0.9500 \\
\hline $\mathrm{C}(6 \mathrm{~S})-\mathrm{H}(73)$ & 0.9500 \\
\hline $\mathrm{C}(22)-\mathrm{Ru}(1)-\mathrm{C}(1)$ & 101.51(15) \\
\hline $\mathrm{C}(22)-\mathrm{Ru}(1)-\mathrm{N}(3)$ & $80.59(14)$ \\
\hline $\mathrm{C}(1)-\mathrm{Ru}(1)-\mathrm{N}(3)$ & $176.33(13)$ \\
\hline $\mathrm{C}(22)-\mathrm{Ru}(1)-\mathrm{Cl}(2)$ & $99.39(12)$ \\
\hline $\mathrm{C}(1)-\mathrm{Ru}(1)-\mathrm{Cl}(2)$ & $89.63(11)$ \\
\hline $\mathrm{N}(3)-\mathrm{Ru}(1)-\mathrm{Cl}(2)$ & $87.05(8)$ \\
\hline $\mathrm{C}(22)-\mathrm{Ru}(1)-\mathrm{Cl}(1)$ & $102.92(12)$ \\
\hline $\mathrm{C}(1)-\mathrm{Ru}(1)-\mathrm{Cl}(1)$ & $95.70(11)$ \\
\hline $\mathrm{N}(3)-\mathrm{Ru}(1)-\mathrm{Cl}(1)$ & $86.71(9)$ \\
\hline $\mathrm{Cl}(2)-\mathrm{Ru}(1)-\mathrm{Cl}(1)$ & $155.50(5)$ \\
\hline $\mathrm{C}(1)-\mathrm{N}(1)-\mathrm{C}(9)$ & $128.6(3)$ \\
\hline $\mathrm{C}(1)-\mathrm{N}(1)-\mathrm{C}(2)$ & $113.9(3)$ \\
\hline $\mathrm{C}(9)-\mathrm{N}(1)-\mathrm{C}(2)$ & $116.3(3)$ \\
\hline
\end{tabular}


Michał Barbasiewicz et al. Structure and Activity Peculiarities of Quinoline and Quinoxaline Ruthenium Complexes...

\begin{tabular}{|c|c|}
\hline$C(1)-N(2)-C(13)$ & $129.8(3)$ \\
\hline $\mathrm{C}(1)-\mathrm{N}(2)-\mathrm{C}(3)$ & $113.9(3)$ \\
\hline $\mathrm{C}(13)-\mathrm{N}(2)-\mathrm{C}(3)$ & $116.3(3)$ \\
\hline $\mathrm{C}(30)-\mathrm{N}(3)-\mathrm{C}(31)$ & $117.7(3)$ \\
\hline $\mathrm{C}(30)-\mathrm{N}(3)-\mathrm{Ru}(1)$ & $130.5(3)$ \\
\hline $\mathrm{C}(31)-\mathrm{N}(3)-\mathrm{Ru}(1)$ & $111.7(2)$ \\
\hline $\mathrm{N}(1)-\mathrm{C}(1)-\mathrm{N}(2)$ & $106.0(3)$ \\
\hline $\mathrm{N}(1)-\mathrm{C}(1)-\mathrm{Ru}(1)$ & $130.7(3)$ \\
\hline $\mathrm{N}(2)-\mathrm{C}(1)-\mathrm{Ru}(1)$ & $122.9(3)$ \\
\hline $\mathrm{N}(1)-\mathrm{C}(2)-\mathrm{C}(3)$ & $102.7(3)$ \\
\hline $\mathrm{N}(1)-\mathrm{C}(2)-\mathrm{H}(2 \mathrm{~A})$ & 111.2 \\
\hline $\mathrm{C}(3)-\mathrm{C}(2)-\mathrm{H}(2 \mathrm{~A})$ & 111.2 \\
\hline $\mathrm{N}(1)-\mathrm{C}(2)-\mathrm{H}(2 \mathrm{~B})$ & 111.2 \\
\hline $\mathrm{C}(3)-\mathrm{C}(2)-\mathrm{H}(2 \mathrm{~B})$ & 111.2 \\
\hline $\mathrm{H}(2 \mathrm{~A})-\mathrm{C}(2)-\mathrm{H}(2 \mathrm{~B})$ & 109.1 \\
\hline$N(2)-C(3)-C(2)$ & $102.5(3)$ \\
\hline $\mathrm{N}(2)-\mathrm{C}(3)-\mathrm{H}(3 \mathrm{~A})$ & 111.3 \\
\hline $\mathrm{C}(2)-\mathrm{C}(3)-\mathrm{H}(3 \mathrm{~A})$ & 111.3 \\
\hline $\mathrm{N}(2)-\mathrm{C}(3)-\mathrm{H}(3 \mathrm{~B})$ & 111.3 \\
\hline $\mathrm{C}(2)-\mathrm{C}(3)-\mathrm{H}(3 \mathrm{~B})$ & 111.3 \\
\hline $\mathrm{H}(3 \mathrm{~A})-\mathrm{C}(3)-\mathrm{H}(3 \mathrm{~B})$ & 109.2 \\
\hline$C(5)-C(4)-C(9)$ & $117.9(4)$ \\
\hline$C(5)-C(4)-C(10)$ & $121.0(4)$ \\
\hline$C(9)-C(4)-C(10)$ & $121.0(4)$ \\
\hline$C(4)-C(5)-C(6)$ & $121.5(4)$ \\
\hline $\mathrm{C}(4)-\mathrm{C}(5)-\mathrm{H}(22)$ & 119.2 \\
\hline $\mathrm{C}(6)-\mathrm{C}(5)-\mathrm{H}(22)$ & 119.2 \\
\hline$C(7)-C(6)-C(5)$ & $118.3(4)$ \\
\hline$C(7)-C(6)-C(11)$ & $121.2(4)$ \\
\hline$C(5)-C(6)-C(11)$ & $120.4(4)$ \\
\hline$C(6)-C(7)-C(8)$ & $122.7(4)$ \\
\hline $\mathrm{C}(6)-\mathrm{C}(7)-\mathrm{H}(48)$ & 118.6 \\
\hline $\mathrm{C}(8)-\mathrm{C}(7)-\mathrm{H}(48)$ & 118.6 \\
\hline $\mathrm{C}(7)-\mathrm{C}(8)-\mathrm{C}(9)$ & $117.3(4)$ \\
\hline$C(7)-C(8)-C(12)$ & $120.5(4)$ \\
\hline$C(9)-C(8)-C(12)$ & $122.1(4)$ \\
\hline $\mathrm{C}(8)-\mathrm{C}(9)-\mathrm{C}(4)$ & $122.1(4)$ \\
\hline $\mathrm{C}(8)-\mathrm{C}(9)-\mathrm{N}(1)$ & $119.0(4)$ \\
\hline $\mathrm{C}(4)-\mathrm{C}(9)-\mathrm{N}(1)$ & 118.7(4) \\
\hline $\mathrm{C}(4)-\mathrm{C}(10)-\mathrm{H}(44 \mathrm{~A})$ & 109.5 \\
\hline $\mathrm{C}(4)-\mathrm{C}(10)-\mathrm{H}(44 \mathrm{~B})$ & 109.5 \\
\hline $\mathrm{H}(44 \mathrm{~A})-\mathrm{C}(10)-\mathrm{H}(44 \mathrm{~B})$ & 109.5 \\
\hline
\end{tabular}


Michał Barbasiewicz et al. Structure and Activity Peculiarities of Quinoline and Quinoxaline Ruthenium Complexes...

\begin{tabular}{|c|c|}
\hline $\mathrm{C}(4)-\mathrm{C}(10)-\mathrm{H}(44 \mathrm{C})$ & 109.5 \\
\hline $\mathrm{H}(44 \mathrm{~A})-\mathrm{C}(10)-\mathrm{H}(44 \mathrm{C})$ & 109.5 \\
\hline $\mathrm{H}(44 \mathrm{~B})-\mathrm{C}(10)-\mathrm{H}(44 \mathrm{C})$ & 109.5 \\
\hline $\mathrm{C}(6)-\mathrm{C}(11)-\mathrm{H}(67 \mathrm{~A})$ & 109.5 \\
\hline $\mathrm{C}(6)-\mathrm{C}(11)-\mathrm{H}(67 \mathrm{~B})$ & 109.5 \\
\hline $\mathrm{H}(67 \mathrm{~A})-\mathrm{C}(11)-\mathrm{H}(67 \mathrm{~B})$ & 109.5 \\
\hline $\mathrm{C}(6)-\mathrm{C}(11)-\mathrm{H}(67 \mathrm{C})$ & 109.5 \\
\hline $\mathrm{H}(67 \mathrm{~A})-\mathrm{C}(11)-\mathrm{H}(67 \mathrm{C})$ & 109.5 \\
\hline $\mathrm{H}(67 \mathrm{~B})-\mathrm{C}(11)-\mathrm{H}(67 \mathrm{C})$ & 109.5 \\
\hline $\mathrm{C}(8)-\mathrm{C}(12)-\mathrm{H}(65 \mathrm{~A})$ & 109.5 \\
\hline $\mathrm{C}(8)-\mathrm{C}(12)-\mathrm{H}(65 \mathrm{~B})$ & 109.5 \\
\hline $\mathrm{H}(65 \mathrm{~A})-\mathrm{C}(12)-\mathrm{H}(65 \mathrm{~B})$ & 109.5 \\
\hline $\mathrm{C}(8)-\mathrm{C}(12)-\mathrm{H}(65 \mathrm{C})$ & 109.5 \\
\hline $\mathrm{H}(65 \mathrm{~A})-\mathrm{C}(12)-\mathrm{H}(65 \mathrm{C})$ & 109.5 \\
\hline $\mathrm{H}(65 \mathrm{~B})-\mathrm{C}(12)-\mathrm{H}(65 \mathrm{C})$ & 109.5 \\
\hline$C(18)-C(13)-C(14)$ & $121.4(4)$ \\
\hline $\mathrm{C}(18)-\mathrm{C}(13)-\mathrm{N}(2)$ & $118.8(4)$ \\
\hline $\mathrm{C}(14)-\mathrm{C}(13)-\mathrm{N}(2)$ & 118.7(4) \\
\hline$C(13)-C(14)-C(15)$ & 118.2(5) \\
\hline$C(13)-C(14)-C(19)$ & $121.6(5)$ \\
\hline$C(15)-C(14)-C(19)$ & 120.1(6) \\
\hline$C(16)-C(15)-C(14)$ & $120.6(6)$ \\
\hline $\mathrm{C}(16)-\mathrm{C}(15)-\mathrm{H}(15)$ & 119.7 \\
\hline $\mathrm{C}(14)-\mathrm{C}(15)-\mathrm{H}(15)$ & 119.7 \\
\hline$C(17)-C(16)-C(15)$ & 121.4(6) \\
\hline$C(17)-C(16)-C(20)$ & $120.3(7)$ \\
\hline$C(15)-C(16)-C(20)$ & 118.2(7) \\
\hline$C(16)-C(17)-C(18)$ & $120.3(6)$ \\
\hline $\mathrm{C}(16)-\mathrm{C}(17)-\mathrm{H}(17)$ & 119.8 \\
\hline $\mathrm{C}(18)-\mathrm{C}(17)-\mathrm{H}(17)$ & 119.8 \\
\hline$C(13)-C(18)-C(17)$ & $117.8(5)$ \\
\hline$C(13)-C(18)-C(21)$ & $123.4(4)$ \\
\hline$C(17)-C(18)-C(21)$ & 118.7(5) \\
\hline $\mathrm{C}(14)-\mathrm{C}(19)-\mathrm{H}(19 \mathrm{~A})$ & 109.5 \\
\hline $\mathrm{C}(14)-\mathrm{C}(19)-\mathrm{H}(19 \mathrm{~B})$ & 109.5 \\
\hline $\mathrm{H}(19 \mathrm{~A})-\mathrm{C}(19)-\mathrm{H}(19 \mathrm{~B})$ & 109.5 \\
\hline $\mathrm{C}(14)-\mathrm{C}(19)-\mathrm{H}(19 \mathrm{C})$ & 109.5 \\
\hline $\mathrm{H}(19 \mathrm{~A})-\mathrm{C}(19)-\mathrm{H}(19 \mathrm{C})$ & 109.5 \\
\hline H(19B)-C(19)-H(19C) & 109.5 \\
\hline $\mathrm{C}(16)-\mathrm{C}(20)-\mathrm{H}(20 \mathrm{~A})$ & 109.5 \\
\hline$C(16)-C(20)-H(20 B)$ & 109.5 \\
\hline $\mathrm{H}(20 \mathrm{~A})-\mathrm{C}(20)-\mathrm{H}(20 \mathrm{~B})$ & 109.5 \\
\hline
\end{tabular}


Michał Barbasiewicz et al. Structure and Activity Peculiarities of Quinoline and Quinoxaline Ruthenium Complexes...

\begin{tabular}{|c|c|}
\hline $\mathrm{C}(16)-\mathrm{C}(20)-\mathrm{H}(20 \mathrm{C})$ & 109.5 \\
\hline $\mathrm{H}(20 \mathrm{~A})-\mathrm{C}(20)-\mathrm{H}(20 \mathrm{C})$ & 109.5 \\
\hline $\mathrm{H}(20 \mathrm{~B})-\mathrm{C}(20)-\mathrm{H}(20 \mathrm{C})$ & 109.5 \\
\hline $\mathrm{C}(18)-\mathrm{C}(21)-\mathrm{H}(21 \mathrm{~A})$ & 109.5 \\
\hline $\mathrm{C}(18)-\mathrm{C}(21)-\mathrm{H}(21 \mathrm{~B})$ & 109.5 \\
\hline $\mathrm{H}(21 \mathrm{~A})-\mathrm{C}(21)-\mathrm{H}(21 \mathrm{~B})$ & 109.5 \\
\hline $\mathrm{C}(18)-\mathrm{C}(21)-\mathrm{H}(21 \mathrm{C})$ & 109.5 \\
\hline $\mathrm{H}(21 \mathrm{~A})-\mathrm{C}(21)-\mathrm{H}(21 \mathrm{C})$ & 109.5 \\
\hline $\mathrm{H}(21 \mathrm{~B})-\mathrm{C}(21)-\mathrm{H}(21 \mathrm{C})$ & 109.5 \\
\hline$C(23)-C(22)-R u(1)$ & $118.4(3)$ \\
\hline $\mathrm{C}(23)-\mathrm{C}(22)-\mathrm{H}(12 \mathrm{~A})$ & 107.7 \\
\hline $\mathrm{Ru}(1)-\mathrm{C}(22)-\mathrm{H}(12 \mathrm{~A})$ & 107.7 \\
\hline $\mathrm{C}(23)-\mathrm{C}(22)-\mathrm{H}(12 \mathrm{~B})$ & 107.7 \\
\hline $\mathrm{Ru}(1)-\mathrm{C}(22)-\mathrm{H}(12 \mathrm{~B})$ & 107.7 \\
\hline $\mathrm{H}(12 \mathrm{~A})-\mathrm{C}(22)-\mathrm{H}(12 \mathrm{~B})$ & 107.1 \\
\hline$C(24)-C(23)-C(31)$ & $118.4(3)$ \\
\hline$C(24)-C(23)-C(22)$ & $127.1(4)$ \\
\hline $\mathrm{C}(31)-\mathrm{C}(23)-\mathrm{C}(22)$ & $114.5(3)$ \\
\hline$C(23)-C(24)-C(25)$ & $120.3(4)$ \\
\hline $\mathrm{C}(23)-\mathrm{C}(24)-\mathrm{H}(30)$ & 119.9 \\
\hline $\mathrm{C}(25)-\mathrm{C}(24)-\mathrm{H}(30)$ & 119.9 \\
\hline$C(26)-C(25)-C(24)$ & $121.0(4)$ \\
\hline $\mathrm{C}(26)-\mathrm{C}(25)-\mathrm{H}(42)$ & 119.5 \\
\hline $\mathrm{C}(24)-\mathrm{C}(25)-\mathrm{H}(42)$ & 119.5 \\
\hline$C(25)-C(26)-C(27)$ & $121.1(4)$ \\
\hline $\mathrm{C}(25)-\mathrm{C}(26)-\mathrm{H}(29)$ & 119.5 \\
\hline $\mathrm{C}(27)-\mathrm{C}(26)-\mathrm{H}(29)$ & 119.5 \\
\hline$C(28)-C(27)-C(31)$ & $116.6(4)$ \\
\hline$C(28)-C(27)-C(26)$ & $126.2(4)$ \\
\hline$C(31)-C(27)-C(26)$ & 117.2(4) \\
\hline$C(29)-C(28)-C(27)$ & $119.2(4)$ \\
\hline $\mathrm{C}(29)-\mathrm{C}(28)-\mathrm{H}(38)$ & 120.4 \\
\hline $\mathrm{C}(27)-\mathrm{C}(28)-\mathrm{H}(38)$ & 120.4 \\
\hline$C(28)-C(29)-C(30)$ & $120.5(4)$ \\
\hline $\mathrm{C}(28)-\mathrm{C}(29)-\mathrm{H}(45)$ & 119.8 \\
\hline $\mathrm{C}(30)-\mathrm{C}(29)-\mathrm{H}(45)$ & 119.8 \\
\hline $\mathrm{N}(3)-\mathrm{C}(30)-\mathrm{C}(29)$ & $122.4(4)$ \\
\hline $\mathrm{N}(3)-\mathrm{C}(30)-\mathrm{H}(37)$ & 118.8 \\
\hline $\mathrm{C}(29)-\mathrm{C}(30)-\mathrm{H}(37)$ & 118.8 \\
\hline $\mathrm{N}(3)-\mathrm{C}(31)-\mathrm{C}(27)$ & $123.6(3)$ \\
\hline$N(3)-C(31)-C(23)$ & $114.5(3)$ \\
\hline$C(27)-C(31)-C(23)$ & $121.9(3)$ \\
\hline
\end{tabular}


Michał Barbasiewicz et al. Structure and Activity Peculiarities of Quinoline and Quinoxaline Ruthenium Complexes. .

$\begin{array}{ll}\mathrm{C}(6 S)-\mathrm{C}(1 S)-\mathrm{C}(2 S) & 119.5(5) \\ \mathrm{C}(6 \mathrm{~S})-\mathrm{C}(1 S)-\mathrm{H}(77) & 120.3 \\ \mathrm{C}(2 S)-\mathrm{C}(1 S)-\mathrm{H}(77) & 120.3 \\ \mathrm{C}(3 S)-\mathrm{C}(2 S)-\mathrm{C}(1 S) & 119.5(6) \\ \mathrm{C}(3 S)-\mathrm{C}(2 S)-\mathrm{H}(74) & 120.3 \\ \mathrm{C}(1 S)-\mathrm{C}(2 S)-\mathrm{H}(74) & 120.3 \\ \mathrm{C}(4 S)-\mathrm{C}(3 S)-\mathrm{C}(2 S) & 121.0(5) \\ \mathrm{C}(4 S)-\mathrm{C}(3 S)-\mathrm{H}(76) & 119.5 \\ \mathrm{C}(2 \mathrm{~S})-\mathrm{C}(3 S)-\mathrm{H}(76) & 119.5 \\ \mathrm{C}(3 S)-\mathrm{C}(4 S)-\mathrm{C}(5 S) & 119.7(5) \\ \mathrm{C}(3 S)-\mathrm{C}(4 S)-\mathrm{H}(75) & 120.1 \\ \mathrm{C}(5 S)-\mathrm{C}(4 S)-\mathrm{H}(75) & 120.1 \\ \mathrm{C}(4 S)-\mathrm{C}(5 S)-\mathrm{C}(6 S) & 119.7(5) \\ \mathrm{C}(4 S)-\mathrm{C}(5 S)-\mathrm{H}(72) & 120.2 \\ \mathrm{C}(6 S)-\mathrm{C}(5 S)-\mathrm{H}(72) & 120.2 \\ \mathrm{C}(1 S)-\mathrm{C}(6 S)-\mathrm{C}(5 S) & 120.7(5) \\ \mathrm{C}(1 S)-\mathrm{C}(6 S)-\mathrm{H}(73) & 119.6 \\ \mathrm{C}(5 S)-\mathrm{C}(6 S)-\mathrm{H}(73) & 119.6 \\ & \end{array}$

Table 4. Anisotropic displacement parameters $\left(\mathrm{A}^{2} \times 10^{3}\right)$ for $\mathrm{C} 003501$ (complex 1a). The anisotropic displacement factor exponent takes the form: $-2 \pi^{2} \times\left[h^{2} \times a^{2} \times U_{11}+\ldots+2 \times h \times k \times a \times b \times U_{12}\right]$

$\begin{array}{lllllll} & \mathbf{U}_{11} & \mathbf{U}_{22} & \mathbf{U}_{33} & \mathbf{U}_{23} & \mathbf{U}_{13} & \mathbf{U}_{12} \\ \mathrm{Ru}(1) & 30(1) & 34(1) & 25(1) & -7(1) & 4(1) & -1(1) \\ \mathrm{Cl}(1) & 49(1) & 41(1) & 37(1) & 1(1) & 3(1) & 5(1) \\ \mathrm{Cl}(2) & 34(1) & 56(1) & 47(1) & -16(1) & -3(1) & 9(1) \\ \mathrm{N}(1) & 42(2) & 42(2) & 33(2) & -18(2) & 14(2) & -16(2) \\ \mathrm{N}(2) & 47(2) & 47(2) & 27(2) & -15(2) & 11(2) & -17(2) \\ \mathrm{N}(3) & 32(2) & 32(2) & 28(2) & -5(1) & 5(1) & -2(1) \\ \mathrm{C}(1) & 32(2) & 42(2) & 29(2) & -5(2) & 10(2) & -2(2) \\ \mathrm{C}(2) & 63(3) & 53(3) & 43(3) & -24(2) & 18(2) & -24(2) \\ \mathrm{C}(3) & 80(4) & 72(3) & 44(3) & -32(2) & 24(3) & -44(3) \\ \mathrm{C}(4) & 36(2) & 37(2) & 34(2) & -6(2) & 5(2) & -10(2) \\ \mathrm{C}(5) & 41(2) & 37(2) & 41(2) & -8(2) & 15(2) & -7(2) \\ \mathrm{C}(6) & 65(3) & 34(2) & 41(2) & -8(2) & 19(2) & -20(2) \\ \mathrm{C}(7) & 64(3) & 33(2) & 48(3) & 3(2) & 12(2) & -5(2) \\ \mathrm{C}(8) & 42(2) & 33(2) & 47(2) & -8(2) & 8(2) & -5(2) \\ \mathrm{C}(9) & 36(2) & 32(2) & 34(2) & -11(2) & 9(2) & -11(2) \\ \mathrm{C}(10) & 43(2) & 62(3) & 43(2) & 4(2) & 3(2) & 3(2) \\ \mathrm{C}(11) & 92(4) & 57(3) & 58(3) & 4(2) & 37(3) & -22(3)\end{array}$


Michał Barbasiewicz et al. Structure and Activity Peculiarities of Quinoline and Quinoxaline Ruthenium Complexes. .

\begin{tabular}{|c|c|c|c|c|c|c|}
\hline & $\mathbf{U}_{11}$ & $\mathbf{U}_{22}$ & $\mathbf{U}_{33}$ & $\mathbf{U}_{23}$ & $\mathbf{U}_{13}$ & $\mathbf{U}_{12}$ \\
\hline$C(12)$ & $59(3)$ & $57(3)$ & $72(3)$ & $-8(3)$ & $11(3)$ & $13(2)$ \\
\hline$C(13)$ & $60(3)$ & $42(2)$ & $23(2)$ & $-11(2)$ & $11(2)$ & $-11(2)$ \\
\hline$C(14)$ & $92(4)$ & $52(3)$ & $33(2)$ & $-14(2)$ & $6(2)$ & $3(3)$ \\
\hline$C(15)$ & $142(5)$ & $40(3)$ & $41(3)$ & $-4(2)$ & $5(3)$ & $-2(3)$ \\
\hline$C(16)$ & $148(5)$ & $51(3)$ & $47(3)$ & $-15(3)$ & $28(3)$ & $-37(4)$ \\
\hline $\mathrm{C}(17)$ & $87(4)$ & $70(3)$ & $54(3)$ & $-28(3)$ & $29(3)$ & $-41(3)$ \\
\hline$C(18)$ & $57(3)$ & $53(3)$ & $34(2)$ & $-19(2)$ & $14(2)$ & $-22(2)$ \\
\hline$C(19)$ & $95(4)$ & $103(4)$ & $57(3)$ & $-18(3)$ & $-14(3)$ & $32(4)$ \\
\hline$C(20)$ & $172(5)$ & $76(3)$ & $68(3)$ & $-2(3)$ & $37(3)$ & $-43(3)$ \\
\hline$C(21)$ & $56(3)$ & $88(4)$ & $56(3)$ & $-20(3)$ & $11(2)$ & $3(3)$ \\
\hline$C(22)$ & $32(2)$ & $28(2)$ & $36(2)$ & $-1(2)$ & $4(2)$ & $5(2)$ \\
\hline$C(23)$ & $39(2)$ & $28(2)$ & $28(2)$ & $1(2)$ & $5(2)$ & $8(2)$ \\
\hline$C(24)$ & $48(2)$ & $33(2)$ & $32(2)$ & $4(2)$ & $11(2)$ & $8(2)$ \\
\hline$C(25)$ & $61(3)$ & $48(2)$ & $24(2)$ & $1(2)$ & $7(2)$ & $17(2)$ \\
\hline$C(26)$ & $53(3)$ & $38(2)$ & $25(2)$ & $-11(2)$ & $-2(2)$ & $9(2)$ \\
\hline$C(27)$ & $38(2)$ & $34(2)$ & $29(2)$ & $-7(2)$ & $-2(2)$ & $6(2)$ \\
\hline$C(28)$ & $38(2)$ & $35(2)$ & $46(3)$ & $-14(2)$ & $-1(2)$ & $-3(2)$ \\
\hline$C(29)$ & $45(3)$ & $38(2)$ & $53(3)$ & $-6(2)$ & $10(2)$ & $-12(2)$ \\
\hline$C(30)$ & $45(2)$ & $43(2)$ & $34(2)$ & $-3(2)$ & $13(2)$ & $-9(2)$ \\
\hline$C(31)$ & $32(2)$ & $27(2)$ & $24(2)$ & $-4(2)$ & $3(2)$ & $4(2)$ \\
\hline $\mathrm{C}(1 \mathrm{~S})$ & $112(5)$ & $49(3)$ & $65(4)$ & $2(3)$ & $11(4)$ & $-4(3)$ \\
\hline$C(2 S)$ & $69(4)$ & $92(5)$ & $68(4)$ & $21(4)$ & $2(3)$ & $13(3)$ \\
\hline$C(3 S)$ & $67(4)$ & $82(4)$ & $45(3)$ & $-4(3)$ & 1(3) & $-25(3)$ \\
\hline $\mathrm{C}(4 \mathrm{~S})$ & $78(4)$ & $58(3)$ & $45(3)$ & $-2(2)$ & $17(3)$ & $-6(3)$ \\
\hline$C(5 S)$ & $59(3)$ & $96(4)$ & $44(3)$ & $17(3)$ & $17(2)$ & $12(3)$ \\
\hline$C(6 S)$ & $68(4)$ & $75(4)$ & $49(3)$ & $6(3)$ & $2(3)$ & $-28(3)$ \\
\hline
\end{tabular}

Table 5. Hydrogen coordinates $\left(\times 10^{4}\right)$ and isotropic displacement parameters $\left(\mathrm{A}^{2} \times 10^{3}\right)$ for C003501 (complex 1a).

$\begin{array}{lllll} & \mathbf{x} & \mathbf{y} & \mathbf{z} & \mathbf{U}(\mathbf{e q}) \\ \mathrm{H}(2 \mathrm{~A}) & 2977 & 4323 & 1416 & 67 \\ \mathrm{H}(2 \mathrm{~B}) & 4315 & 3426 & 1689 & 67 \\ \mathrm{H}(3 \mathrm{~A}) & 5528 & 4143 & 916 & 83 \\ \mathrm{H}(3 \mathrm{~B}) & 4080 & 4821 & 542 & 83 \\ \mathrm{H}(22) & 1271 & 6147 & 3670 & 49 \\ \mathrm{H}(48) & 4460 & 3775 & 4399 & 59 \\ \mathrm{H}(44 \mathrm{~A}) & 1110 & 7054 & 2542 & 77 \\ \mathrm{H}(44 \mathrm{~B}) & 1983 & 6377 & 1940 & 77\end{array}$


Michał Barbasiewicz et al. Structure and Activity Peculiarities of Quinoline and Quinoxaline Ruthenium Complexes. .

\begin{tabular}{|c|c|c|c|c|}
\hline & $\mathbf{x}$ & $\mathbf{y}$ & $\mathbf{z}$ & $\mathrm{U}(\mathrm{eq})$ \\
\hline $\mathrm{H}(44 \mathrm{C})$ & 2685 & 7629 & 2569 & 77 \\
\hline $\mathrm{H}(67 \mathrm{~A})$ & 2647 & 4122 & 5081 & 100 \\
\hline $\mathrm{H}(67 \mathrm{~B})$ & 1094 & 4281 & 4555 & 100 \\
\hline $\mathrm{H}(67 \mathrm{C})$ & 1987 & 5557 & 5086 & 100 \\
\hline $\mathrm{H}(65 \mathrm{~A})$ & 5707 & 2978 & 2871 & 99 \\
\hline $\mathrm{H}(65 \mathrm{~B})$ & 6313 & 3223 & 3774 & 99 \\
\hline $\mathrm{H}(65 \mathrm{C})$ & 6712 & 4269 & 3287 & 99 \\
\hline $\mathrm{H}(15)$ & 5351 & 8868 & -232 & 94 \\
\hline $\mathrm{H}(17)$ & 9028 & 7043 & 367 & 87 \\
\hline $\mathrm{H}(19 \mathrm{~A})$ & 3245 & 6982 & 544 & 141 \\
\hline $\mathrm{H}(19 \mathrm{~B})$ & 3227 & 8191 & 90 & 141 \\
\hline $\mathrm{H}(19 \mathrm{C})$ & 3626 & 8464 & 1001 & 141 \\
\hline $\mathrm{H}(20 \mathrm{~A})$ & 7177 & 8836 & -980 & 159 \\
\hline $\mathrm{H}(20 \mathrm{~B})$ & 8719 & 8277 & -667 & 159 \\
\hline $\mathrm{H}(20 \mathrm{C})$ & 8280 & 9673 & -260 & 159 \\
\hline $\mathrm{H}(21 \mathrm{~A})$ & 7968 & 5337 & 1711 & 106 \\
\hline $\mathrm{H}(21 \mathrm{~B})$ & 9341 & 5776 & 1432 & 106 \\
\hline $\mathrm{H}(21 \mathrm{C})$ & 8211 & 4655 & 900 & 106 \\
\hline $\mathrm{H}(12 \mathrm{~A})$ & 4687 & 8059 & 3648 & 40 \\
\hline $\mathrm{H}(12 \mathrm{~B})$ & 5830 & 6981 & 3870 & 40 \\
\hline $\mathrm{H}(30)$ & 5375 & 8242 & 5242 & 46 \\
\hline $\mathrm{H}(42)$ & 6639 & 9769 & 6304 & 55 \\
\hline $\mathrm{H}(29)$ & 8339 & 11271 & 6181 & 51 \\
\hline $\mathrm{H}(38)$ & 9694 & 12240 & 5279 & 53 \\
\hline $\mathrm{H}(45)$ & 10020 & 12082 & 4051 & 57 \\
\hline $\mathrm{H}(37)$ & 8763 & 10439 & 3074 & 50 \\
\hline $\mathrm{H}(77)$ & 9607 & 3688 & 2555 & 93 \\
\hline $\mathrm{H}(74)$ & 7549 & 2463 & 1794 & 93 \\
\hline $\mathrm{H}(76)$ & 7723 & 250 & 1286 & 82 \\
\hline $\mathrm{H}(75)$ & 9889 & -771 & 1541 & 73 \\
\hline $\mathrm{H}(72)$ & 11943 & 448 & 2285 & 78 \\
\hline $\mathrm{H}(73)$ & 11780 & 2667 & 2799 & 80 \\
\hline
\end{tabular}

Table 6. Torsion angles [deg] for C003501 (complex 1a).

$$
\begin{array}{ll}
\mathrm{C}(22)-\mathrm{Ru}(1)-\mathrm{N}(3)-\mathrm{C}(30) & -177.5(4) \\
\mathrm{C}(1)-\mathrm{Ru}(1)-\mathrm{N}(3)-\mathrm{C}(30) & 57(2) \\
\mathrm{Cl}(2)-\mathrm{Ru}(1)-\mathrm{N}(3)-\mathrm{C}(30) & 82.5(3) \\
\mathrm{Cl}(1)-\mathrm{Ru}(1)-\mathrm{N}(3)-\mathrm{C}(30) & -73.8(3)
\end{array}
$$


Michał Barbasiewicz et al. Structure and Activity Peculiarities of Quinoline and Quinoxaline Ruthenium Complexes...

\begin{tabular}{|c|c|}
\hline $\mathrm{C}(22)-\mathrm{Ru}(1)-\mathrm{N}(3)-\mathrm{C}(31)$ & $4.3(2)$ \\
\hline $\mathrm{C}(1)-\mathrm{Ru}(1)-\mathrm{N}(3)-\mathrm{C}(31)$ & $-121(2)$ \\
\hline $\mathrm{Cl}(2)-\mathrm{Ru}(1)-\mathrm{N}(3)-\mathrm{C}(31)$ & $-95.7(2)$ \\
\hline $\mathrm{Cl}(1)-\mathrm{Ru}(1)-\mathrm{N}(3)-\mathrm{C}(31)$ & $108.0(2)$ \\
\hline $\mathrm{C}(9)-\mathrm{N}(1)-\mathrm{C}(1)-\mathrm{N}(2)$ & $-170.0(4)$ \\
\hline $\mathrm{C}(2)-\mathrm{N}(1)-\mathrm{C}(1)-\mathrm{N}(2)$ & $-3.3(5)$ \\
\hline $\mathrm{C}(9)-\mathrm{N}(1)-\mathrm{C}(1)-\mathrm{Ru}(1)$ & $17.4(6)$ \\
\hline $\mathrm{C}(2)-\mathrm{N}(1)-\mathrm{C}(1)-\mathrm{Ru}(1)$ & $-175.9(3)$ \\
\hline $\mathrm{C}(13)-\mathrm{N}(2)-\mathrm{C}(1)-\mathrm{N}(1)$ & $179.7(4)$ \\
\hline $\mathrm{C}(3)-\mathrm{N}(2)-\mathrm{C}(1)-\mathrm{N}(1)$ & $-3.8(5)$ \\
\hline $\mathrm{C}(13)-\mathrm{N}(2)-\mathrm{C}(1)-\mathrm{Ru}(1)$ & $-7.1(6)$ \\
\hline $\mathrm{C}(3)-\mathrm{N}(2)-\mathrm{C}(1)-\mathrm{Ru}(1)$ & $169.5(3)$ \\
\hline $\mathrm{C}(22)-\mathrm{Ru}(1)-\mathrm{C}(1)-\mathrm{N}(1)$ & $-12.8(4)$ \\
\hline $\mathrm{N}(3)-\mathrm{Ru}(1)-\mathrm{C}(1)-\mathrm{N}(1)$ & $112(2)$ \\
\hline $\mathrm{Cl}(2)-\mathrm{Ru}(1)-\mathrm{C}(1)-\mathrm{N}(1)$ & $86.7(4)$ \\
\hline $\mathrm{Cl}(1)-\mathrm{Ru}(1)-\mathrm{C}(1)-\mathrm{N}(1)$ & $-117.3(4)$ \\
\hline $\mathrm{C}(22)-\mathrm{Ru}(1)-\mathrm{C}(1)-\mathrm{N}(2)$ & $175.7(3)$ \\
\hline $\mathrm{N}(3)-\mathrm{Ru}(1)-\mathrm{C}(1)-\mathrm{N}(2)$ & $-60(2)$ \\
\hline $\mathrm{Cl}(2)-\mathrm{Ru}(1)-\mathrm{C}(1)-\mathrm{N}(2)$ & $-84.8(3)$ \\
\hline $\mathrm{Cl}(1)-\mathrm{Ru}(1)-\mathrm{C}(1)-\mathrm{N}(2)$ & $71.3(3)$ \\
\hline$C(1)-N(1)-C(2)-C(3)$ & $8.6(5)$ \\
\hline$C(9)-N(1)-C(2)-C(3)$ & $177.0(4)$ \\
\hline $\mathrm{C}(1)-\mathrm{N}(2)-\mathrm{C}(3)-\mathrm{C}(2)$ & $8.8(6)$ \\
\hline $\mathrm{C}(13)-\mathrm{N}(2)-\mathrm{C}(3)-\mathrm{C}(2)$ & $-174.1(4)$ \\
\hline $\mathrm{N}(1)-\mathrm{C}(2)-\mathrm{C}(3)-\mathrm{N}(2)$ & $-9.6(5)$ \\
\hline$C(9)-C(4)-C(5)-C(6)$ & $0.0(5)$ \\
\hline$C(10)-C(4)-C(5)-C(6)$ & $-177.3(4)$ \\
\hline$C(4)-C(5)-C(6)-C(7)$ & $0.8(6)$ \\
\hline$C(4)-C(5)-C(6)-C(11)$ & $-178.4(4)$ \\
\hline$C(5)-C(6)-C(7)-C(8)$ & $-2.4(6)$ \\
\hline$C(11)-C(6)-C(7)-C(8)$ & $176.9(4)$ \\
\hline$C(6)-C(7)-C(8)-C(9)$ & $2.9(6)$ \\
\hline$C(6)-C(7)-C(8)-C(12)$ & $-176.1(4)$ \\
\hline$C(7)-C(8)-C(9)-C(4)$ & $-2.0(6)$ \\
\hline $\mathrm{C}(12)-\mathrm{C}(8)-\mathrm{C}(9)-\mathrm{C}(4)$ & $177.0(4)$ \\
\hline $\mathrm{C}(7)-\mathrm{C}(8)-\mathrm{C}(9)-\mathrm{N}(1)$ & $-177.4(3)$ \\
\hline $\mathrm{C}(12)-\mathrm{C}(8)-\mathrm{C}(9)-\mathrm{N}(1)$ & $1.6(6)$ \\
\hline$C(5)-C(4)-C(9)-C(8)$ & $0.6(5)$ \\
\hline $\mathrm{C}(10)-\mathrm{C}(4)-\mathrm{C}(9)-\mathrm{C}(8)$ & $177.9(3)$ \\
\hline$C(5)-C(4)-C(9)-N(1)$ & $176.0(3)$ \\
\hline $\mathrm{C}(10)-\mathrm{C}(4)-\mathrm{C}(9)-\mathrm{N}(1)$ & $-6.7(5)$ \\
\hline $\mathrm{C}(1)-\mathrm{N}(1)-\mathrm{C}(9)-\mathrm{C}(8)$ & $-107.5(5)$ \\
\hline
\end{tabular}


Michał Barbasiewicz et al. Structure and Activity Peculiarities of Quinoline and Quinoxaline Ruthenium Complexes...

\begin{tabular}{|c|c|}
\hline $\mathrm{C}(2)-\mathrm{N}(1)-\mathrm{C}(9)-\mathrm{C}(8)$ & $86.0(5)$ \\
\hline $\mathrm{C}(1)-\mathrm{N}(1)-\mathrm{C}(9)-\mathrm{C}(4)$ & $76.9(5)$ \\
\hline$C(2)-N(1)-C(9)-C(4)$ & $-89.5(5)$ \\
\hline $\mathrm{C}(1)-\mathrm{N}(2)-\mathrm{C}(13)-\mathrm{C}(18)$ & $97.1(5)$ \\
\hline $\mathrm{C}(3)-\mathrm{N}(2)-\mathrm{C}(13)-\mathrm{C}(18)$ & $-79.4(5)$ \\
\hline $\mathrm{C}(1)-\mathrm{N}(2)-\mathrm{C}(13)-\mathrm{C}(14)$ & $-94.7(5)$ \\
\hline $\mathrm{C}(3)-\mathrm{N}(2)-\mathrm{C}(13)-\mathrm{C}(14)$ & $88.8(5)$ \\
\hline $\mathrm{C}(18)-\mathrm{C}(13)-\mathrm{C}(14)-\mathrm{C}(15)$ & $-5.5(6)$ \\
\hline $\mathrm{N}(2)-\mathrm{C}(13)-\mathrm{C}(14)-\mathrm{C}(15)$ & $-173.4(4)$ \\
\hline C(18)-C(13)-C(14)-C(19) & $170.9(4)$ \\
\hline $\mathrm{N}(2)-\mathrm{C}(13)-\mathrm{C}(14)-\mathrm{C}(19)$ & $3.0(6)$ \\
\hline $\mathrm{C}(13)-\mathrm{C}(14)-\mathrm{C}(15)-\mathrm{C}(16)$ & $1.3(7)$ \\
\hline$C(19)-C(14)-C(15)-C(16)$ & $-175.1(5)$ \\
\hline $\mathrm{C}(14)-\mathrm{C}(15)-\mathrm{C}(16)-\mathrm{C}(17)$ & $2.0(8)$ \\
\hline $\mathrm{C}(14)-\mathrm{C}(15)-\mathrm{C}(16)-\mathrm{C}(20)$ & $-179.7(5)$ \\
\hline $\mathrm{C}(15)-\mathrm{C}(16)-\mathrm{C}(17)-\mathrm{C}(18)$ & $-1.1(8)$ \\
\hline $\mathrm{C}(20)-\mathrm{C}(16)-\mathrm{C}(17)-\mathrm{C}(18)$ & $-179.5(4)$ \\
\hline $\mathrm{C}(14)-\mathrm{C}(13)-\mathrm{C}(18)-\mathrm{C}(17)$ & $6.3(6)$ \\
\hline $\mathrm{N}(2)-\mathrm{C}(13)-\mathrm{C}(18)-\mathrm{C}(17)$ & 174.1(3) \\
\hline $\mathrm{C}(14)-\mathrm{C}(13)-\mathrm{C}(18)-\mathrm{C}(21)$ & $-169.9(4)$ \\
\hline $\mathrm{N}(2)-\mathrm{C}(13)-\mathrm{C}(18)-\mathrm{C}(21)$ & $-2.1(6)$ \\
\hline $\mathrm{C}(16)-\mathrm{C}(17)-\mathrm{C}(18)-\mathrm{C}(13)$ & $-2.9(6)$ \\
\hline $\mathrm{C}(16)-\mathrm{C}(17)-\mathrm{C}(18)-\mathrm{C}(21)$ & $173.4(4)$ \\
\hline$C(1)-R u(1)-C(22)-C(23)$ & 172.1(3) \\
\hline$N(3)-R u(1)-C(22)-C(23)$ & $-4.8(3)$ \\
\hline $\mathrm{Cl}(2)-\mathrm{Ru}(1)-\mathrm{C}(22)-\mathrm{C}(23)$ & $80.6(3)$ \\
\hline $\mathrm{Cl}(1)-\mathrm{Ru}(1)-\mathrm{C}(22)-\mathrm{C}(23)$ & $-89.2(3)$ \\
\hline $\mathrm{Ru}(1)-\mathrm{C}(22)-\mathrm{C}(23)-\mathrm{C}(24)$ & $-176.6(3)$ \\
\hline $\mathrm{Ru}(1)-\mathrm{C}(22)-\mathrm{C}(23)-\mathrm{C}(31)$ & $4.8(4)$ \\
\hline$C(31)-C(23)-C(24)-C(25)$ & $-1.9(5)$ \\
\hline$C(22)-C(23)-C(24)-C(25)$ & $179.5(4)$ \\
\hline $\mathrm{C}(23)-\mathrm{C}(24)-\mathrm{C}(25)-\mathrm{C}(26)$ & $1.6(6)$ \\
\hline$C(24)-C(25)-C(26)-C(27)$ & $0.3(6)$ \\
\hline$C(25)-C(26)-C(27)-C(28)$ & $178.8(4)$ \\
\hline $\mathrm{C}(25)-\mathrm{C}(26)-\mathrm{C}(27)-\mathrm{C}(31)$ & $-1.7(6)$ \\
\hline $\mathrm{C}(31)-\mathrm{C}(27)-\mathrm{C}(28)-\mathrm{C}(29)$ & $0.3(6)$ \\
\hline $\mathrm{C}(26)-\mathrm{C}(27)-\mathrm{C}(28)-\mathrm{C}(29)$ & $179.8(4)$ \\
\hline $\mathrm{C}(27)-\mathrm{C}(28)-\mathrm{C}(29)-\mathrm{C}(30)$ & $-1.0(6)$ \\
\hline $\mathrm{C}(31)-\mathrm{N}(3)-\mathrm{C}(30)-\mathrm{C}(29)$ & $0.5(6)$ \\
\hline $\mathrm{Ru}(1)-\mathrm{N}(3)-\mathrm{C}(30)-\mathrm{C}(29)$ & $-177.6(3)$ \\
\hline $\mathrm{C}(28)-\mathrm{C}(29)-\mathrm{C}(30)-\mathrm{N}(3)$ & $0.6(7)$ \\
\hline $\mathrm{C}(30)-\mathrm{N}(3)-\mathrm{C}(31)-\mathrm{C}(27)$ & $-1.2(5)$ \\
\hline
\end{tabular}


Michał Barbasiewicz et al. Structure and Activity Peculiarities of Quinoline and Quinoxaline Ruthenium Complexes...

$\begin{array}{ll}\mathrm{Ru}(1)-\mathrm{N}(3)-\mathrm{C}(31)-\mathrm{C}(27) & 177.2(3) \\ \mathrm{C}(30)-\mathrm{N}(3)-\mathrm{C}(31)-\mathrm{C}(23) & 178.7(3) \\ \mathrm{Ru}(1)-\mathrm{N}(3)-\mathrm{C}(31)-\mathrm{C}(23) & -2.9(4) \\ \mathrm{C}(28)-\mathrm{C}(27)-\mathrm{C}(31)-\mathrm{N}(3) & 0.8(5) \\ \mathrm{C}(26)-\mathrm{C}(27)-\mathrm{C}(31)-\mathrm{N}(3) & -178.7(3) \\ \mathrm{C}(28)-\mathrm{C}(27)-\mathrm{C}(31)-\mathrm{C}(23) & -179.1(3) \\ \mathrm{C}(26)-\mathrm{C}(27)-\mathrm{C}(31)-\mathrm{C}(23) & 1.4(5) \\ \mathrm{C}(24)-\mathrm{C}(23)-\mathrm{C}(31)-\mathrm{N}(3) & -179.5(3) \\ \mathrm{C}(22)-\mathrm{C}(23)-\mathrm{C}(31)-\mathrm{N}(3) & -0.7(5) \\ \mathrm{C}(24)-\mathrm{C}(23)-\mathrm{C}(31)-\mathrm{C}(27) & 0.4(5) \\ \mathrm{C}(22)-\mathrm{C}(23)-\mathrm{C}(31)-\mathrm{C}(27) & 179.2(3) \\ \mathrm{C}(6 \mathrm{~S})-\mathrm{C}(1 \mathrm{~S})-\mathrm{C}(2 \mathrm{~S})-\mathrm{C}(3 \mathrm{~S}) & -0.2(9) \\ \mathrm{C}(1 \mathrm{~S})-\mathrm{C}(2 \mathrm{~S})-\mathrm{C}(3 \mathrm{~S})-\mathrm{C}(4 S) & 0.6(9) \\ \mathrm{C}(2 \mathrm{~S})-\mathrm{C}(3 \mathrm{~S})-\mathrm{C}(4 \mathrm{~S})-\mathrm{C}(5 \mathrm{~S}) & -1.0(8) \\ \mathrm{C}(3 \mathrm{~S})-\mathrm{C}(4 S)-\mathrm{C}(5 \mathrm{~S})-\mathrm{C}(6 \mathrm{~S}) & 1.0(8) \\ \mathrm{C}(2 \mathrm{~S})-\mathrm{C}(1 \mathrm{~S})-\mathrm{C}(6 \mathrm{~S})-\mathrm{C}(5 \mathrm{~S}) & 0.3(8) \\ \mathrm{C}(4 \mathrm{~S})-\mathrm{C}(5 \mathrm{~S})-\mathrm{C}(6 \mathrm{~S})-\mathrm{C}(1 S) & -0.7(8)\end{array}$


Figure 2. ORTEP drawing of C0083 (cis-dichloro, complex 1b) represented by thermal ellipsoids drawn at the $50 \%$ probability level.

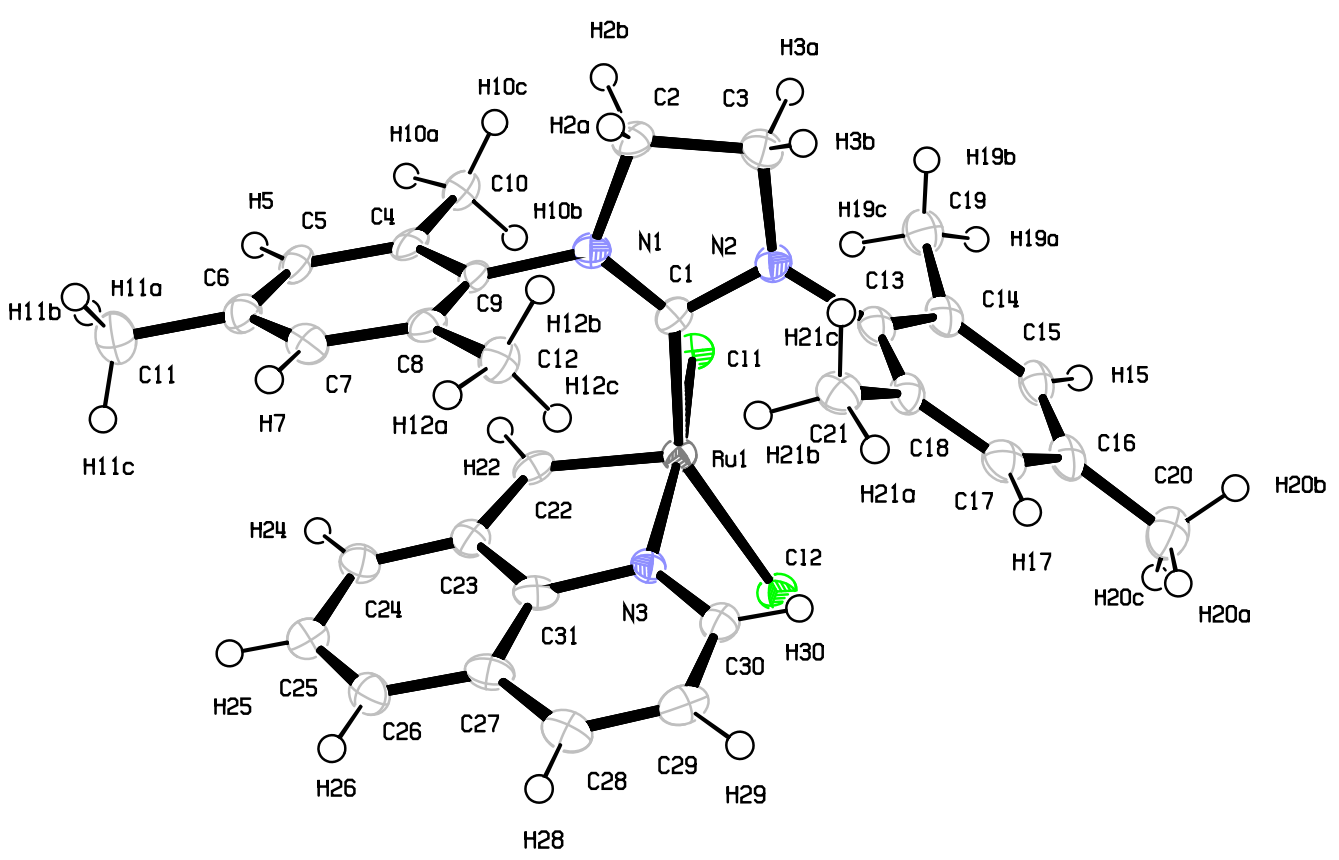

Table 7. Crystal data and structure refinement for C0083 (complex 1b).

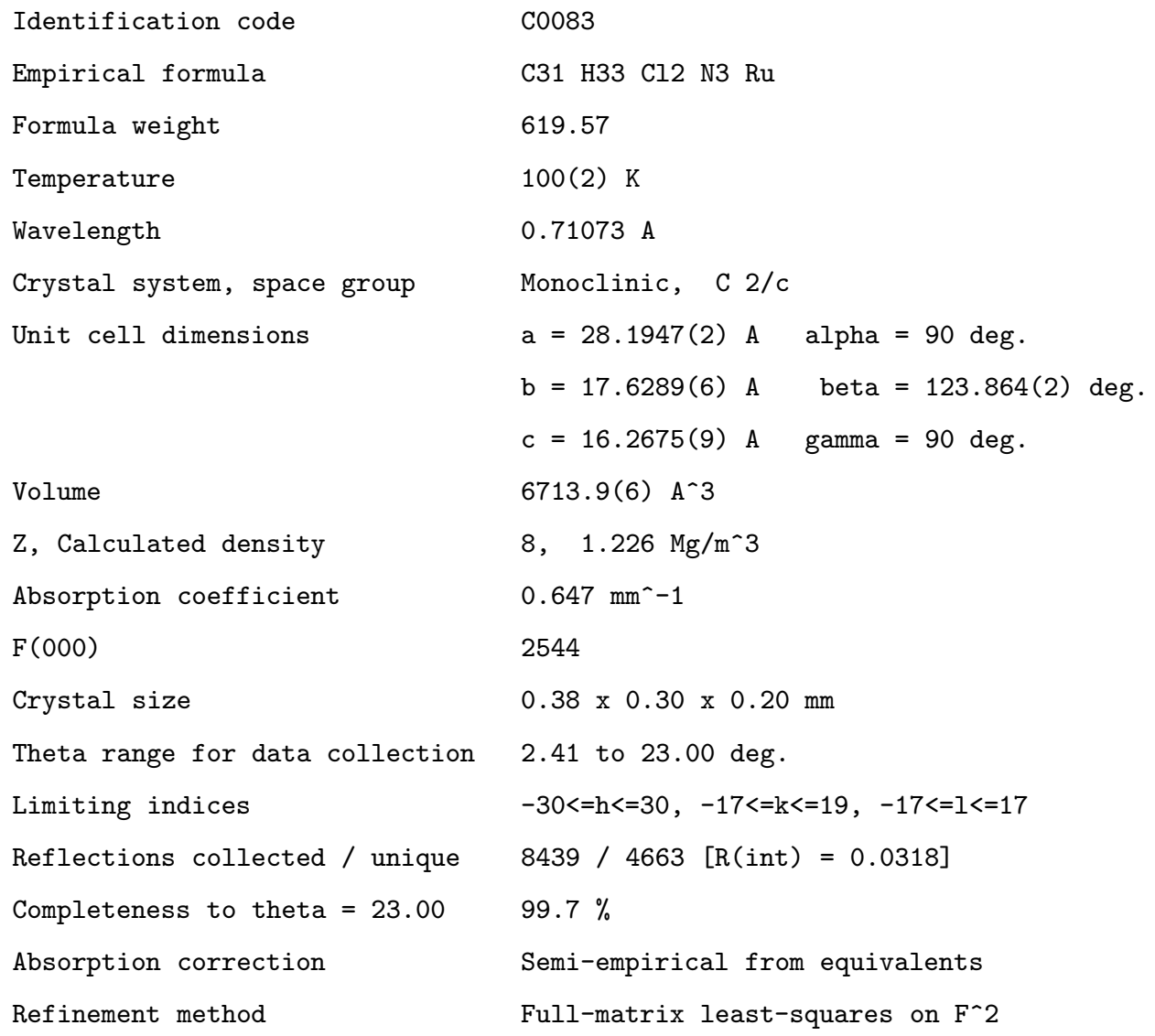


Michał Barbasiewicz et al. Structure and Activity Peculiarities of Quinoline and Quinoxaline Ruthenium Complexes. .

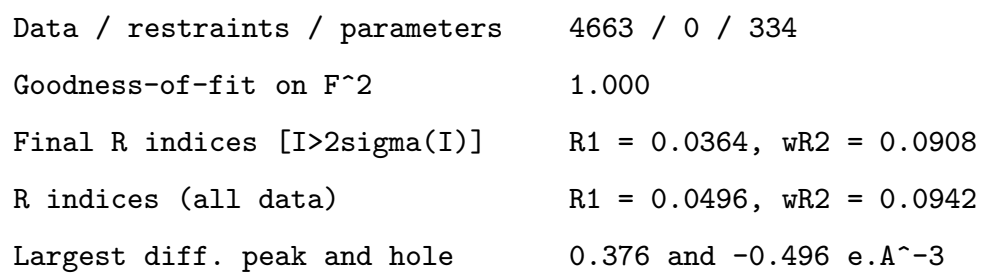

Table 8. Atomic coordinates $\left(\times 10^{4}\right)$ and equivalent isotropic displacement parameters $\left(\mathrm{A}^{2} \times\right.$ $10^{3}$ ) for $\mathrm{C} 0083$ (complex 1b). $\mathrm{U}(\mathrm{eq})$ is defined as one third of the trace of the orthogonalized $\mathrm{U}_{i j}$ tensor.

$\begin{array}{lllll} & \mathbf{x} & \mathbf{y} & \mathbf{z} & \mathbf{U}(\mathbf{e q}) \\ \mathrm{C}(1) & 2114(2) & 562(2) & 8578(3) & 18(1) \\ \mathrm{C}(2) & 2482(2) & 1259(2) & 10051(3) & 22(1) \\ \mathrm{C}(3) & 2665(2) & 432(2) & 10328(3) & 26(1) \\ \mathrm{C}(4) & 2146(2) & 2436(2) & 8175(3) & 20(1) \\ \mathrm{C}(5) & 1869(2) & 3112(2) & 7697(3) & 21(1) \\ \mathrm{C}(6) & 1329(2) & 3290(2) & 7472(3) & 23(1) \\ \mathrm{C}(7) & 1062(2) & 2777(2) & 7739(3) & 22(1) \\ \mathrm{C}(8) & 1315(2) & 2091(2) & 8214(3) & 20(1) \\ \mathrm{C}(9) & 1854(2) & 1928(2) & 8402(3) & 19(1) \\ \mathrm{C}(10) & 2741(2) & 2261(2) & 8450(3) & 24(1) \\ \mathrm{C}(11) & 1027(2) & 4020(2) & 6940(3) & 33(1) \\ \mathrm{C}(12) & 1011(2) & 1559(2) & 8489(3) & 24(1) \\ \mathrm{C}(13) & 2308(2) & -744(2) & 9178(3) & 22(1) \\ \mathrm{C}(14) & 2716(2) & -1207(2) & 9210(3) & 24(1) \\ \mathrm{C}(15) & 2579(2) & -1973(2) & 8973(3) & 24(1) \\ \mathrm{C}(16) & 2071(2) & -2280(2) & 8746(3) & 25(1) \\ \mathrm{C}(17) & 1695(2) & -1807(2) & 8813(3) & 26(1) \\ \mathrm{C}(18) & 1807(2) & -1041(2) & 9039(3) & 21(1) \\ \mathrm{C}(19) & 3302(2) & -911(2) & 9554(3) & 29(1) \\ \mathrm{C}(20) & 1923(2) & -3107(2) & 8465(3) & 34(1) \\ \mathrm{C}(21) & 1411(2) & -564(2) & 9173(3) & 25(1) \\ \mathrm{C}(22) & 1599(2) & 1075(2) & 6593(3) & 20(1) \\ \mathrm{C}(23) & 1014(2) & 1311(2) & 6116(3) & 22(1) \\ \mathrm{C}(24) & 747(2) & 1962(2) & 5583(3) & 25(1) \\ \mathrm{C}(25) & 165(2) & 2094(2) & 5170(3) & 30(1) \\ \mathrm{C}(26) & -160(2) & 1569(2) & 5267(3) & 25(1) \\ \mathrm{C}(27) & 89(2) & 880(2) & 5794(3) & 24(1) \\ \mathrm{C}(28) & -217(2) & 286(2) & 5889(3) & 25(1) \\ \mathrm{C}(29) & 73(2) & -360(2) & 6370(3) & 26(1) \\ & & & & \end{array}$


Michał Barbasiewicz et al. Structure and Activity Peculiarities of Quinoline and Quinoxaline Ruthenium Complexes. .

$\begin{array}{lllll} & \mathbf{x} & \mathbf{y} & \mathbf{z} & \mathbf{U}(\mathbf{e q}) \\ \mathrm{C}(30) & 657(2) & -431(2) & 6772(3) & 21(1) \\ \mathrm{C}(31) & 670(2) & 1250(2) & 6214(3) & 21(1) \\ \mathrm{N}(1) & 2137(1) & 66(2) & 8947(2) & 21(1) \\ \mathrm{N}(2) & 124(2) & 9349(2) & 20(1) \\ \mathrm{N}(3) & 2405(1) & 281(1) & 6728(2) & 18(1) \\ \mathrm{Cl}(1) & 962(1) & -873(1) & 7551(1) & 23(1) \\ \mathrm{Cl}(2) & 2748(1) & 159(1) & 6144(1) & 23(1) \\ \operatorname{Ru}(1) & 1635(1) & & 7219(1) & 17(1)\end{array}$

Table 9. Bond lengths $[\AA \mathrm{A}]$ and angles [deg] for C0083 (complex 1b).

$\begin{array}{ll}\mathrm{C}(1)-\mathrm{N}(1) & 1.338(5) \\ \mathrm{C}(1)-\mathrm{N}(2) & 1.365(5) \\ \mathrm{C}(1)-\mathrm{Ru}(1) & 2.008(4) \\ \mathrm{C}(2)-\mathrm{N}(1) & 1.491(5) \\ \mathrm{C}(2)-\mathrm{C}(3) & 1.527(5) \\ \mathrm{C}(2)-\mathrm{H}(2 \mathrm{~A}) & 0.9900 \\ \mathrm{C}(2)-\mathrm{H}(2 \mathrm{~B}) & 0.9900 \\ \mathrm{C}(3)-\mathrm{N}(2) & 1.480(5) \\ \mathrm{C}(3)-\mathrm{H}(3 \mathrm{~A}) & 0.9900 \\ \mathrm{C}(3)-\mathrm{H}(3 \mathrm{~B}) & 0.9900 \\ \mathrm{C}(4)-\mathrm{C}(5) & 1.399(5) \\ \mathrm{C}(4)-\mathrm{C}(9) & 1.399(5) \\ \mathrm{C}(4)-\mathrm{C}(10) & 1.508(5) \\ \mathrm{C}(5)-\mathrm{C}(6) & 1.390(6) \\ \mathrm{C}(5)-\mathrm{H}(5) & 0.9500 \\ \mathrm{C}(6)-\mathrm{C}(7) & 1.392(5) \\ \mathrm{C}(6)-\mathrm{C}(11) & 1.519(5) \\ \mathrm{C}(7)-\mathrm{C}(8) & 1.397(5) \\ \mathrm{C}(7)-\mathrm{H}(7) & 0.9500 \\ \mathrm{C}(8)-\mathrm{C}(9) & 1.401(6) \\ \mathrm{C}(8)-\mathrm{C}(12) & 1.499(5) \\ \mathrm{C}(9)-\mathrm{N}(1) & 1.438(5) \\ \mathrm{C}(10)-\mathrm{H}(10 \mathrm{~A}) & 0.9800 \\ \mathrm{C}(10)-\mathrm{H}(10 \mathrm{~B}) & 0.9800 \\ \mathrm{C}(10)-\mathrm{H}(10 \mathrm{C}) & 0.9800 \\ \mathrm{C}(11)-\mathrm{H}(11 \mathrm{~A}) & 0.9800 \\ \mathrm{C}(11)-\mathrm{H}(11 \mathrm{~B}) & 0.9800 \\ \mathrm{C}(11)-\mathrm{H}(11 \mathrm{C}) & \\ & 0.900 \\ & \\ & \\ & \end{array}$


Michał Barbasiewicz et al. Structure and Activity Peculiarities of Quinoline and Quinoxaline Ruthenium Complexes...

\begin{tabular}{|c|c|}
\hline $\mathrm{C}(12)-\mathrm{H}(12 \mathrm{~A})$ & 0.9800 \\
\hline $\mathrm{C}(12)-\mathrm{H}(12 \mathrm{~B})$ & 0.9800 \\
\hline $\mathrm{C}(12)-\mathrm{H}(12 \mathrm{C})$ & 0.9800 \\
\hline$C(13)-C(14)$ & $1.388(5)$ \\
\hline$C(13)-C(18)$ & $1.402(5)$ \\
\hline $\mathrm{C}(13)-\mathrm{N}(2)$ & $1.451(5)$ \\
\hline$C(14)-C(15)$ & $1.399(5)$ \\
\hline$C(14)-C(19)$ & $1.510(6)$ \\
\hline$C(15)-C(16)$ & $1.374(6)$ \\
\hline $\mathrm{C}(15)-\mathrm{H}(15)$ & 0.9500 \\
\hline$C(16)-C(17)$ & $1.400(6)$ \\
\hline$C(16)-C(20)$ & $1.515(5)$ \\
\hline$C(17)-C(18)$ & $1.389(5)$ \\
\hline $\mathrm{C}(17)-\mathrm{H}(17)$ & 0.9500 \\
\hline$C(18)-C(21)$ & $1.508(5)$ \\
\hline C(19)-H(19A) & 0.9800 \\
\hline C(19)-H(19B) & 0.9800 \\
\hline C(19)-H(19C) & 0.9800 \\
\hline $\mathrm{C}(20)-\mathrm{H}(20 \mathrm{~A})$ & 0.9800 \\
\hline $\mathrm{C}(20)-\mathrm{H}(20 \mathrm{~B})$ & 0.9800 \\
\hline $\mathrm{C}(20)-\mathrm{H}(20 \mathrm{C})$ & 0.9800 \\
\hline $\mathrm{C}(21)-\mathrm{H}(21 \mathrm{~A})$ & 0.9800 \\
\hline $\mathrm{C}(21)-\mathrm{H}(21 \mathrm{~B})$ & 0.9800 \\
\hline $\mathrm{C}(21)-\mathrm{H}(21 \mathrm{C})$ & 0.9800 \\
\hline$C(22)-C(23)$ & $1.439(6)$ \\
\hline $\mathrm{C}(22)-\mathrm{Ru}(1)$ & $1.823(4)$ \\
\hline $\mathrm{C}(22)-\mathrm{H}(22)$ & 0.9500 \\
\hline$C(23)-C(24)$ & $1.381(5)$ \\
\hline$C(23)-C(31)$ & $1.431(5)$ \\
\hline$C(24)-C(25)$ & $1.404(6)$ \\
\hline $\mathrm{C}(24)-\mathrm{H}(24)$ & 0.9500 \\
\hline$C(25)-C(26)$ & $1.372(6)$ \\
\hline $\mathrm{C}(25)-\mathrm{H}(25)$ & 0.9500 \\
\hline$C(26)-C(27)$ & $1.426(6)$ \\
\hline $\mathrm{C}(26)-\mathrm{H}(26)$ & 0.9500 \\
\hline$C(27)-C(31)$ & $1.393(6)$ \\
\hline$C(27)-C(28)$ & $1.420(6)$ \\
\hline$C(28)-C(29)$ & $1.366(6)$ \\
\hline $\mathrm{C}(28)-\mathrm{H}(28)$ & 0.9500 \\
\hline C(29)-C(30) & $1.398(6)$ \\
\hline C(29)-H(29) & 0.9500 \\
\hline $\mathrm{C}(30)-\mathrm{N}(3)$ & $1.330(5)$ \\
\hline
\end{tabular}


Michał Barbasiewicz et al. Structure and Activity Peculiarities of Quinoline and Quinoxaline Ruthenium Complexes...

\begin{tabular}{|c|c|}
\hline $\mathrm{C}(30)-\mathrm{H}(30)$ & 0.9500 \\
\hline $\mathrm{C}(31)-\mathrm{N}(3)$ & $1.380(5)$ \\
\hline $\mathrm{N}(3)-\mathrm{Ru}(1)$ & $2.072(3)$ \\
\hline $\mathrm{Cl}(1)-\mathrm{Ru}(1)$ & $2.3812(10)$ \\
\hline $\mathrm{Cl}(2)-\mathrm{Ru}(1)$ & $2.3731(10)$ \\
\hline $\mathrm{N}(1)-\mathrm{C}(1)-\mathrm{N}(2)$ & $108.4(3)$ \\
\hline $\mathrm{N}(1)-\mathrm{C}(1)-\mathrm{Ru}(1)$ & $134.5(3)$ \\
\hline $\mathrm{N}(2)-\mathrm{C}(1)-\mathrm{Ru}(1)$ & $116.9(2)$ \\
\hline $\mathrm{N}(1)-\mathrm{C}(2)-\mathrm{C}(3)$ & $103.4(3)$ \\
\hline $\mathrm{N}(1)-\mathrm{C}(2)-\mathrm{H}(2 \mathrm{~A})$ & 111.1 \\
\hline $\mathrm{C}(3)-\mathrm{C}(2)-\mathrm{H}(2 \mathrm{~A})$ & 111.1 \\
\hline $\mathrm{N}(1)-\mathrm{C}(2)-\mathrm{H}(2 \mathrm{~B})$ & 111.1 \\
\hline $\mathrm{C}(3)-\mathrm{C}(2)-\mathrm{H}(2 \mathrm{~B})$ & 111.1 \\
\hline $\mathrm{H}(2 \mathrm{~A})-\mathrm{C}(2)-\mathrm{H}(2 \mathrm{~B})$ & 109.0 \\
\hline $\mathrm{N}(2)-\mathrm{C}(3)-\mathrm{C}(2)$ & $102.4(3)$ \\
\hline $\mathrm{N}(2)-\mathrm{C}(3)-\mathrm{H}(3 \mathrm{~A})$ & 111.3 \\
\hline $\mathrm{C}(2)-\mathrm{C}(3)-\mathrm{H}(3 \mathrm{~A})$ & 111.3 \\
\hline $\mathrm{N}(2)-\mathrm{C}(3)-\mathrm{H}(3 \mathrm{~B})$ & 111.3 \\
\hline $\mathrm{C}(2)-\mathrm{C}(3)-\mathrm{H}(3 \mathrm{~B})$ & 111.3 \\
\hline $\mathrm{H}(3 \mathrm{~A})-\mathrm{C}(3)-\mathrm{H}(3 \mathrm{~B})$ & 109.2 \\
\hline$C(5)-C(4)-C(9)$ & $117.6(4)$ \\
\hline$C(5)-C(4)-C(10)$ & $121.5(3)$ \\
\hline$C(9)-C(4)-C(10)$ & $120.8(3)$ \\
\hline$C(6)-C(5)-C(4)$ & $121.9(4)$ \\
\hline $\mathrm{C}(6)-\mathrm{C}(5)-\mathrm{H}(5)$ & 119.0 \\
\hline $\mathrm{C}(4)-\mathrm{C}(5)-\mathrm{H}(5)$ & 119.0 \\
\hline$C(5)-C(6)-C(7)$ & $118.4(4)$ \\
\hline$C(5)-C(6)-C(11)$ & $121.7(4)$ \\
\hline$C(7)-C(6)-C(11)$ & $120.0(4)$ \\
\hline$C(6)-C(7)-C(8)$ & $122.4(4)$ \\
\hline $\mathrm{C}(6)-\mathrm{C}(7)-\mathrm{H}(7)$ & 118.8 \\
\hline $\mathrm{C}(8)-\mathrm{C}(7)-\mathrm{H}(7)$ & 118.8 \\
\hline$C(7)-C(8)-C(9)$ & 117.1(3) \\
\hline$C(7)-C(8)-C(12)$ & $120.4(4)$ \\
\hline$C(9)-C(8)-C(12)$ & $122.5(3)$ \\
\hline$C(4)-C(9)-C(8)$ & $122.5(3)$ \\
\hline $\mathrm{C}(4)-\mathrm{C}(9)-\mathrm{N}(1)$ & 119.2(4) \\
\hline $\mathrm{C}(8)-\mathrm{C}(9)-\mathrm{N}(1)$ & $118.0(3)$ \\
\hline $\mathrm{C}(4)-\mathrm{C}(10)-\mathrm{H}(10 \mathrm{~A})$ & 109.5 \\
\hline $\mathrm{C}(4)-\mathrm{C}(10)-\mathrm{H}(10 \mathrm{~B})$ & 109.5 \\
\hline $\mathrm{H}(10 \mathrm{~A})-\mathrm{C}(10)-\mathrm{H}(10 \mathrm{~B})$ & 109.5 \\
\hline
\end{tabular}


Michał Barbasiewicz et al. Structure and Activity Peculiarities of Quinoline and Quinoxaline Ruthenium Complexes...

\begin{tabular}{|c|c|}
\hline $\mathrm{C}(4)-\mathrm{C}(10)-\mathrm{H}(10 \mathrm{C})$ & 109.5 \\
\hline $\mathrm{H}(10 \mathrm{~A})-\mathrm{C}(10)-\mathrm{H}(10 \mathrm{C})$ & 109.5 \\
\hline $\mathrm{H}(10 \mathrm{~B})-\mathrm{C}(10)-\mathrm{H}(10 \mathrm{C})$ & 109.5 \\
\hline $\mathrm{C}(6)-\mathrm{C}(11)-\mathrm{H}(11 \mathrm{~A})$ & 109.5 \\
\hline $\mathrm{C}(6)-\mathrm{C}(11)-\mathrm{H}(11 \mathrm{~B})$ & 109.5 \\
\hline $\mathrm{H}(11 \mathrm{~A})-\mathrm{C}(11)-\mathrm{H}(11 \mathrm{~B})$ & 109.5 \\
\hline $\mathrm{C}(6)-\mathrm{C}(11)-\mathrm{H}(11 \mathrm{C})$ & 109.5 \\
\hline $\mathrm{H}(11 \mathrm{~A})-\mathrm{C}(11)-\mathrm{H}(11 \mathrm{C})$ & 109.5 \\
\hline $\mathrm{H}(11 \mathrm{~B})-\mathrm{C}(11)-\mathrm{H}(11 \mathrm{C})$ & 109.5 \\
\hline $\mathrm{C}(8)-\mathrm{C}(12)-\mathrm{H}(12 \mathrm{~A})$ & 109.5 \\
\hline $\mathrm{C}(8)-\mathrm{C}(12)-\mathrm{H}(12 \mathrm{~B})$ & 109.5 \\
\hline $\mathrm{H}(12 \mathrm{~A})-\mathrm{C}(12)-\mathrm{H}(12 \mathrm{~B})$ & 109.5 \\
\hline $\mathrm{C}(8)-\mathrm{C}(12)-\mathrm{H}(12 \mathrm{C})$ & 109.5 \\
\hline $\mathrm{H}(12 \mathrm{~A})-\mathrm{C}(12)-\mathrm{H}(12 \mathrm{C})$ & 109.5 \\
\hline $\mathrm{H}(12 \mathrm{~B})-\mathrm{C}(12)-\mathrm{H}(12 \mathrm{C})$ & 109.5 \\
\hline$C(14)-C(13)-C(18)$ & $121.8(3)$ \\
\hline $\mathrm{C}(14)-\mathrm{C}(13)-\mathrm{N}(2)$ & $120.4(4)$ \\
\hline $\mathrm{C}(18)-\mathrm{C}(13)-\mathrm{N}(2)$ & 117.7(3) \\
\hline$C(13)-C(14)-C(15)$ & $117.4(4)$ \\
\hline$C(13)-C(14)-C(19)$ & $121.6(3)$ \\
\hline$C(15)-C(14)-C(19)$ & $120.9(4)$ \\
\hline$C(16)-C(15)-C(14)$ & $122.8(4)$ \\
\hline $\mathrm{C}(16)-\mathrm{C}(15)-\mathrm{H}(15)$ & 118.6 \\
\hline $\mathrm{C}(14)-\mathrm{C}(15)-\mathrm{H}(15)$ & 118.6 \\
\hline$C(15)-C(16)-C(17)$ & $117.7(4)$ \\
\hline$C(15)-C(16)-C(20)$ & $122.1(4)$ \\
\hline$C(17)-C(16)-C(20)$ & $120.2(4)$ \\
\hline$C(18)-C(17)-C(16)$ & $122.1(4)$ \\
\hline $\mathrm{C}(18)-\mathrm{C}(17)-\mathrm{H}(17)$ & 119.0 \\
\hline $\mathrm{C}(16)-\mathrm{C}(17)-\mathrm{H}(17)$ & 119.0 \\
\hline$C(17)-C(18)-C(13)$ & $117.7(4)$ \\
\hline$C(17)-C(18)-C(21)$ & $120.3(4)$ \\
\hline$C(13)-C(18)-C(21)$ & $122.0(3)$ \\
\hline $\mathrm{C}(14)-\mathrm{C}(19)-\mathrm{H}(19 \mathrm{~A})$ & 109.5 \\
\hline $\mathrm{C}(14)-\mathrm{C}(19)-\mathrm{H}(19 \mathrm{~B})$ & 109.5 \\
\hline H(19A)-C(19)-H(19B) & 109.5 \\
\hline $\mathrm{C}(14)-\mathrm{C}(19)-\mathrm{H}(19 \mathrm{C})$ & 109.5 \\
\hline $\mathrm{H}(19 \mathrm{~A})-\mathrm{C}(19)-\mathrm{H}(19 \mathrm{C})$ & 109.5 \\
\hline H(19B)-C(19)-H(19C) & 109.5 \\
\hline $\mathrm{C}(16)-\mathrm{C}(20)-\mathrm{H}(20 \mathrm{~A})$ & 109.5 \\
\hline$C(16)-C(20)-H(20 B)$ & 109.5 \\
\hline $\mathrm{H}(20 \mathrm{~A})-\mathrm{C}(20)-\mathrm{H}(20 \mathrm{~B})$ & 109.5 \\
\hline
\end{tabular}


Michał Barbasiewicz et al. Structure and Activity Peculiarities of Quinoline and Quinoxaline Ruthenium Complexes...

\begin{tabular}{|c|c|}
\hline $\mathrm{C}(16)-\mathrm{C}(20)-\mathrm{H}(20 \mathrm{C})$ & 109.5 \\
\hline $\mathrm{H}(20 \mathrm{~A})-\mathrm{C}(20)-\mathrm{H}(20 \mathrm{C})$ & 109.5 \\
\hline $\mathrm{H}(20 \mathrm{~B})-\mathrm{C}(20)-\mathrm{H}(20 \mathrm{C})$ & 109.5 \\
\hline $\mathrm{C}(18)-\mathrm{C}(21)-\mathrm{H}(21 \mathrm{~A})$ & 109.5 \\
\hline $\mathrm{C}(18)-\mathrm{C}(21)-\mathrm{H}(21 \mathrm{~B})$ & 109.5 \\
\hline $\mathrm{H}(21 \mathrm{~A})-\mathrm{C}(21)-\mathrm{H}(21 \mathrm{~B})$ & 109.5 \\
\hline $\mathrm{C}(18)-\mathrm{C}(21)-\mathrm{H}(21 \mathrm{C})$ & 109.5 \\
\hline $\mathrm{H}(21 \mathrm{~A})-\mathrm{C}(21)-\mathrm{H}(21 \mathrm{C})$ & 109.5 \\
\hline $\mathrm{H}(21 \mathrm{~B})-\mathrm{C}(21)-\mathrm{H}(21 \mathrm{C})$ & 109.5 \\
\hline$C(23)-C(22)-R u(1)$ & $118.9(3)$ \\
\hline $\mathrm{C}(23)-\mathrm{C}(22)-\mathrm{H}(22)$ & 120.5 \\
\hline $\mathrm{Ru}(1)-\mathrm{C}(22)-\mathrm{H}(22)$ & 120.5 \\
\hline$C(24)-C(23)-C(31)$ & 117.2(4) \\
\hline$C(24)-C(23)-C(22)$ & $129.7(4)$ \\
\hline$C(31)-C(23)-C(22)$ & $113.0(3)$ \\
\hline$C(23)-C(24)-C(25)$ & $121.4(4)$ \\
\hline $\mathrm{C}(23)-\mathrm{C}(24)-\mathrm{H}(24)$ & 119.3 \\
\hline $\mathrm{C}(25)-\mathrm{C}(24)-\mathrm{H}(24)$ & 119.3 \\
\hline$C(26)-C(25)-C(24)$ & $120.8(4)$ \\
\hline $\mathrm{C}(26)-\mathrm{C}(25)-\mathrm{H}(25)$ & 119.6 \\
\hline $\mathrm{C}(24)-\mathrm{C}(25)-\mathrm{H}(25)$ & 119.6 \\
\hline$C(25)-C(26)-C(27)$ & $120.4(4)$ \\
\hline $\mathrm{C}(25)-\mathrm{C}(26)-\mathrm{H}(26)$ & 119.8 \\
\hline $\mathrm{C}(27)-\mathrm{C}(26)-\mathrm{H}(26)$ & 119.8 \\
\hline$C(31)-C(27)-C(28)$ & $117.7(4)$ \\
\hline$C(31)-C(27)-C(26)$ & $117.7(4)$ \\
\hline$C(28)-C(27)-C(26)$ & $124.6(4)$ \\
\hline$C(29)-C(28)-C(27)$ & $118.0(4)$ \\
\hline $\mathrm{C}(29)-\mathrm{C}(28)-\mathrm{H}(28)$ & 121.0 \\
\hline $\mathrm{C}(27)-\mathrm{C}(28)-\mathrm{H}(28)$ & 121.0 \\
\hline$C(28)-C(29)-C(30)$ & 121.1(4) \\
\hline $\mathrm{C}(28)-\mathrm{C}(29)-\mathrm{H}(29)$ & 119.4 \\
\hline $\mathrm{C}(30)-\mathrm{C}(29)-\mathrm{H}(29)$ & 119.4 \\
\hline$N(3)-C(30)-C(29)$ & $122.4(4)$ \\
\hline $\mathrm{N}(3)-\mathrm{C}(30)-\mathrm{H}(30)$ & 118.8 \\
\hline $\mathrm{C}(29)-\mathrm{C}(30)-\mathrm{H}(30)$ & 118.8 \\
\hline $\mathrm{N}(3)-\mathrm{C}(31)-\mathrm{C}(27)$ & $123.5(4)$ \\
\hline$N(3)-C(31)-C(23)$ & $114.0(3)$ \\
\hline$C(27)-C(31)-C(23)$ & $122.5(4)$ \\
\hline $\mathrm{C}(1)-\mathrm{N}(1)-\mathrm{C}(9)$ & 127.3(3) \\
\hline $\mathrm{C}(1)-\mathrm{N}(1)-\mathrm{C}(2)$ & 112.7(3) \\
\hline $\mathrm{C}(9)-\mathrm{N}(1)-\mathrm{C}(2)$ & 119.9(3) \\
\hline
\end{tabular}


Michał Barbasiewicz et al. Structure and Activity Peculiarities of Quinoline and Quinoxaline Ruthenium Complexes. .

$\begin{array}{ll}\mathrm{C}(1)-\mathrm{N}(2)-\mathrm{C}(13) & 120.2(3) \\ \mathrm{C}(1)-\mathrm{N}(2)-\mathrm{C}(3) & 113.1(3) \\ \mathrm{C}(13)-\mathrm{N}(2)-\mathrm{C}(3) & 124.2(3) \\ \mathrm{C}(30)-\mathrm{N}(3)-\mathrm{C}(31) & 117.1(3) \\ \mathrm{C}(30)-\mathrm{N}(3)-\mathrm{Ru}(1) & 130.3(3) \\ \mathrm{C}(31)-\mathrm{N}(3)-\mathrm{Ru}(1) & 112.4(2) \\ \mathrm{C}(22)-\mathrm{Ru}(1)-\mathrm{C}(1) & 96.08(15) \\ \mathrm{C}(22)-\mathrm{Ru}(1)-\mathrm{N}(3) & 81.73(15) \\ \mathrm{C}(1)-\mathrm{Ru}(1)-\mathrm{N}(3) & 96.82(13) \\ \mathrm{C}(22)-\mathrm{Ru}(1)-\mathrm{Cl}(2) & 113.56(12) \\ \mathrm{C}(1)-\mathrm{Ru}(1)-\mathrm{Cl}(2) & 150.36(11) \\ \mathrm{N}(3)-\mathrm{Ru}(1)-\mathrm{Cl}(2) & 88.15(8) \\ \mathrm{C}(22)-\mathrm{Ru}(1)-\mathrm{Cl}(1) & 91.69(13) \\ \mathrm{C}(1)-\mathrm{Ru}(1)-\mathrm{Cl}(1) & 89.16(11) \\ \mathrm{N}(3)-\mathrm{Ru}(1)-\mathrm{Cl}(1) & 171.52(9) \\ \mathrm{Cl}(2)-\mathrm{Ru}(1)-\mathrm{Cl}(1) & 89.61(3)\end{array}$

Table 10. Anisotropic displacement parameters $\left(\mathrm{A}^{2} \times 10^{3}\right)$ for C0083 (complex 1b). The anisotropic displacement factor exponent takes the form: $-2 \pi^{2} \times\left[h^{2} \times a^{2} \times U_{11}+\ldots+2 \times h \times k \times a \times b \times U_{12}\right]$

$\begin{array}{lllllll} & \mathbf{U}_{11} & \mathbf{U}_{22} & \mathbf{U}_{33} & \mathbf{U}_{23} & \mathbf{U}_{13} & \mathbf{U}_{12} \\ \mathrm{C}(1) & 20(2) & 18(2) & 22(2) & -2(2) & 15(2) & -4(2) \\ \mathrm{C}(2) & 30(3) & 22(2) & 17(2) & -5(2) & 15(2) & -4(2) \\ \mathrm{C}(3) & 36(3) & 27(2) & 17(2) & 0(2) & 15(2) & 1(2) \\ \mathrm{C}(4) & 32(3) & 17(2) & 14(2) & -7(2) & 14(2) & -6(2) \\ \mathrm{C}(5) & 32(3) & 15(2) & 20(2) & -2(2) & 17(2) & -2(2) \\ \mathrm{C}(6) & 28(3) & 20(2) & 21(3) & -2(2) & 14(2) & 0(2) \\ \mathrm{C}(7) & 22(2) & 27(2) & 20(2) & -1(2) & 13(2) & 3(2) \\ \mathrm{C}(8) & 24(3) & 20(2) & 16(2) & -5(2) & 11(2) & -5(2) \\ \mathrm{C}(9) & 25(3) & 12(2) & 15(2) & -3(2) & 9(2) & -2(2) \\ \mathrm{C}(10) & 28(3) & 19(2) & 27(3) & -4(2) & 17(2) & -2(2) \\ \mathrm{C}(11) & 32(3) & 28(2) & 38(3) & 8(2) & 20(3) & 5(2) \\ \mathrm{C}(12) & 29(3) & 22(2) & 25(3) & 1(2) & 18(2) & -1(2) \\ \mathrm{C}(13) & 29(3) & 23(2) & 15(2) & 2(2) & 13(2) & 3(2) \\ \mathrm{C}(14) & 34(3) & 26(2) & 16(2) & 4(2) & 17(2) & 4(2) \\ \mathrm{C}(15) & 34(3) & 22(2) & 21(3) & 4(2) & 18(2) & 8(2) \\ \mathrm{C}(16) & 40(3) & 23(2) & 17(2) & 4(2) & 19(2) & 8(2) \\ \mathrm{C}(17) & 28(3) & 31(2) & 20(3) & 3(2) & 14(2) & -1(2) \\ \mathrm{C}(18) & 27(3) & 19(2) & 19(2) & 2(2) & 14(2) & 3(2) \\ \mathrm{C}(19) & 31(3) & 29(2) & 31(3) & 3(2) & 19(2) & 4(2)\end{array}$


Michał Barbasiewicz et al. Structure and Activity Peculiarities of Quinoline and Quinoxaline Ruthenium Complexes...

$\begin{array}{lllllll} & \mathbf{U}_{11} & \mathbf{U}_{22} & \mathbf{U}_{33} & \mathbf{U}_{23} & \mathbf{U}_{13} & \mathbf{U}_{12} \\ \mathrm{C}(20) & 54(3) & 26(2) & 32(3) & -1(2) & 30(3) & -2(2) \\ \mathrm{C}(21) & 27(3) & 30(2) & 20(3) & -1(2) & 14(2) & 2(2) \\ \mathrm{C}(22) & 24(3) & 21(2) & 20(2) & -8(2) & 15(2) & -6(2) \\ \mathrm{C}(23) & 29(3) & 23(2) & 16(2) & -1(2) & 14(2) & -2(2) \\ \mathrm{C}(24) & 28(3) & 26(2) & 20(3) & 0(2) & 12(2) & 1(2) \\ \mathrm{C}(25) & 29(3) & 25(2) & 28(3) & -2(2) & 11(2) & 0(2) \\ \mathrm{C}(26) & 25(3) & 26(2) & 23(3) & -3(2) & 12(2) & 7(2) \\ \mathrm{C}(27) & 19(3) & 35(2) & 17(2) & -4(2) & 10(2) & -2(2) \\ \mathrm{C}(28) & 24(2) & 37(3) & 19(2) & -1(2) & 14(2) & 2(2) \\ \mathrm{C}(29) & 30(3) & 31(2) & 22(3) & -6(2) & 18(2) & -11(2) \\ \mathrm{C}(30) & 27(3) & 21(2) & 18(2) & -2(2) & 14(2) & -2(2) \\ \mathrm{C}(31) & 25(3) & 30(2) & 10(2) & -4(2) & 11(2) & -3(2) \\ \mathrm{N}(1) & 25(2) & 20(2) & 16(2) & -2(1) & 11(2) & 0(2) \\ \mathrm{N}(2) & 26(2) & 19(2) & 19(2) & -1(1) & 14(2) & 1(2) \\ \mathrm{N}(3) & 21(2) & 18(2) & 13(2) & 0(1) & 9(2) & 1(2) \\ \mathrm{Cl}(1) & 21(1) & 27(1) & 23(1) & -3(1) & 14(1) & -2(1) \\ \mathrm{Cl}(2) & 24(1) & 23(1) & 22(1) & -5(1) & 13(1) & -1(1) \\ \mathrm{Ru}(1) & 19(1) & 19(1) & 16(1) & -1(1) & 11(1) & 0(1)\end{array}$

Table 11. Hydrogen coordinates $\left(\times 10^{4}\right)$ and isotropic displacement parameters $\left(\mathrm{A}^{2} \times 10^{3}\right)$ for C0083 (complex 1b).

$\begin{array}{lllll} & \mathbf{x} & \mathbf{y} & \mathbf{z} & \mathbf{U}(\mathbf{e q}) \\ \mathrm{H}(2 \mathrm{~A}) & 2250 & 1429 & 10300 & 26 \\ \mathrm{H}(2 \mathrm{~B}) & 2818 & 1595 & 10320 & 26 \\ \mathrm{H}(3 \mathrm{~A}) & 3086 & 382 & 10729 & 32 \\ \mathrm{H}(3 \mathrm{~B}) & 2513 & 213 & 10698 & 32 \\ \mathrm{H}(5) & 2056 & 3460 & 7522 & 25 \\ \mathrm{H}(7) & 694 & 2898 & 7594 & 27 \\ \mathrm{H}(10 \mathrm{~A}) & 2876 & 2677 & 8230 & 36 \\ \mathrm{H}(10 B) & 2736 & 1789 & 8128 & 36 \\ \mathrm{H}(10 \mathrm{C}) & 2997 & 2206 & 9170 & 36 \\ \mathrm{H}(11 \mathrm{~A}) & 1272 & 4313 & 6804 & 49 \\ \mathrm{H}(11 \mathrm{~B}) & 949 & 4320 & 7359 & 49 \\ \mathrm{H}(11 \mathrm{C}) & 667 & 3899 & 6316 & 49 \\ \mathrm{H}(12 \mathrm{~A}) & 643 & 1779 & 8293 & 36 \\ \mathrm{H}(12 B) & 1244 & 1476 & 9207 & 36 \\ \mathrm{H}(12 \mathrm{C}) & 947 & 1074 & 8148 & 36 \\ \mathrm{H}(15) & 2848 & -2295 & 8969 & 29\end{array}$


Michał Barbasiewicz et al. Structure and Activity Peculiarities of Quinoline and Quinoxaline Ruthenium Complexes...

$\begin{array}{lllll} & \mathbf{x} & \mathbf{y} & \mathbf{z} & \mathbf{U}(\mathbf{e q}) \\ \mathrm{H}(17) & 1353 & -2016 & 8700 & 31 \\ \mathrm{H}(19 \mathrm{~A}) & 3527 & -1319 & 9521 & 43 \\ \mathrm{H}(19 B) & 3495 & -731 & 10238 & 43 \\ \mathrm{H}(19 \mathrm{C}) & 3264 & -491 & 9126 & 43 \\ \mathrm{H}(20 \mathrm{~A}) & 1545 & -3213 & 8332 & 50 \\ \mathrm{H}(20 B) & 2209 & -3428 & 9011 & 50 \\ \mathrm{H}(20 \mathrm{C}) & 1920 & -3215 & 7871 & 50 \\ \mathrm{H}(21 \mathrm{~A}) & 1086 & -873 & 9042 & 38 \\ \mathrm{H}(21 \mathrm{~B}) & 1271 & -135 & 8711 & 38 \\ \mathrm{H}(21 \mathrm{C}) & 1617 & -373 & 9854 & 38 \\ \mathrm{H}(22) & 1869 & 1390 & 6581 & 24 \\ \mathrm{H}(24) & 962 & 2328 & 5493 & 30 \\ \mathrm{H}(25) & -5 & 2552 & 4820 & 36 \\ \mathrm{H}(26) & -554 & 1666 & 4982 & 30 \\ \mathrm{H}(28) & -613 & 337 & 5627 & 30 \\ \mathrm{H}(29) & -125 & -768 & 6431 & 31 \\ \mathrm{H}(30) & 843 & -893 & 7089 & 25\end{array}$




\section{Charges and electron density cubes}

Figure 3. Optimized structures of 8-vinylquinoline and 5-vinylquinoxaline and their calculated ESP charges.
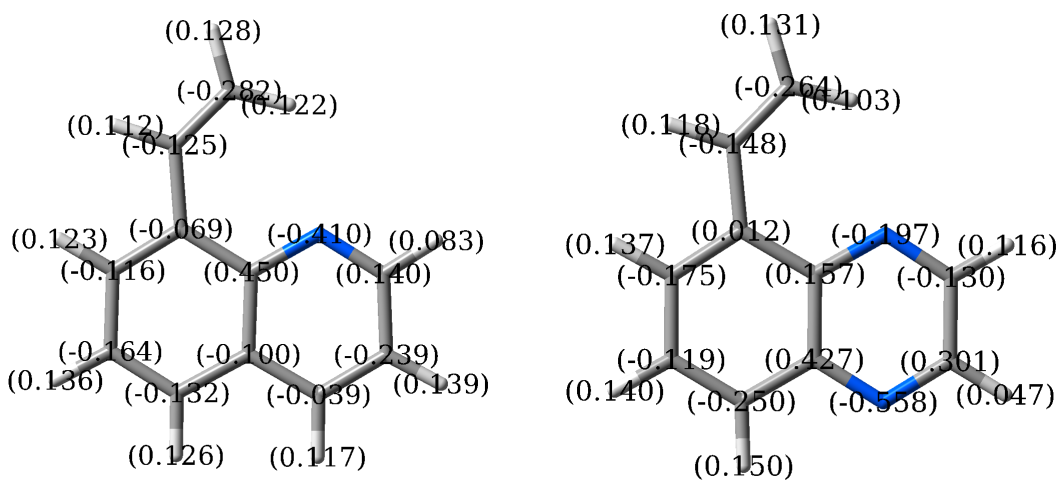

Figure 4. Optimized structures of 8-vinylquinoline and 5-vinylquinoxaline and their electron density cubes mapped with ESP.

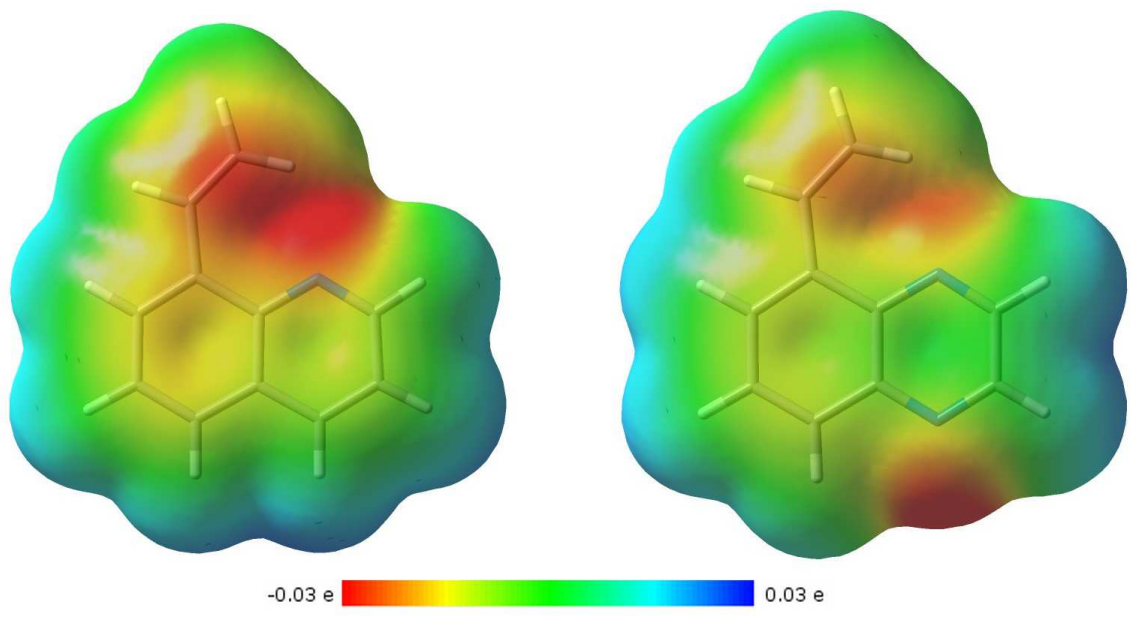

All the calculations were performed using Gaussian $03 .{ }^{3}$ The structures of 8-vinylquinoline and 5-vinylquinoxaline were optimized using B3LYP with the $6-31 \mathrm{G}^{* *}$ basis set in a gas phase. Only real values of the analytical harmonic vibrational frequencies confirmed that the geometries under study correspond to the minimum energy structures.

${ }^{3}$ Gaussian 03, Revision B.05, Frisch, M. J.; Trucks, G. W.; Schlegel, H. B.; Scuseria, G. E.; Robb, M. A.; Cheeseman, J. R.; Montgomery, Jr., J. A.; Vreven, T.; Kudin, K. N.; Burant, J. C.; Millam, J. M.; Iyengar, S. S.; Tomasi, J.; Barone, V.; Mennucci, B.; Cossi, M.; Scalmani, G.; Rega, N.; Petersson, G. A.; Nakatsuji, H.; Hada, M.; Ehara, M.; Toyota, K.; Fukuda, R.; Hasegawa, J.; Ishida, M.; Nakajima, T.; Honda, Y.; Kitao, O.; Nakai, H.; Klene, M.; Li, X.; Knox, J. E.; Hratchian, H. P.; Cross, J. B.; Adamo, C.; Jaramillo, J.; Gomperts, R.; Stratmann, R. E.; Yazyev, O.; Austin, A. J.; Cammi, R.; Pomelli, C.; Ochterski, J. W.; Ayala, P. Y.; Morokuma, K.; Voth, G. A.; Salvador, P.; Dannenberg, J. J.; Zakrzewski, V. G.; Dapprich, S.; Daniels, A. D.; Strain, M. C.; Farkas, O.; Malick, D. K.; Rabuck, A. D.; Raghavachari, K.; Foresman, J. B.; Ortiz, J. V.; Cui, Q.; Baboul, A. G.; Clifford, S.; Cioslowski, J.; Stefanov, B. B.; Liu, G.; Liashenko, A.; Piskorz, P.; Komaromi, I.; Martin, R. L.; Fox, D. J.; Keith, T.; Al-Laham, M. A.; Peng, C. Y.; Nanayakkara, A.; Challacombe, M.; Gill, P. M. W.; Johnson, B.; Chen, W.; Wong, M. W.; Gonzalez, C. and Pople, J. A. Gaussian, Inc., Pittsburgh PA, 2003. 
Michał Barbasiewicz et al. Structure and Activity Peculiarities of Quinoline and Quinoxaline Ruthenium Complexes...

\section{NMR and ESI Spectra}

\section{5-Vinylquinaxoline $-{ }^{1} \mathrm{H}$ NMR}

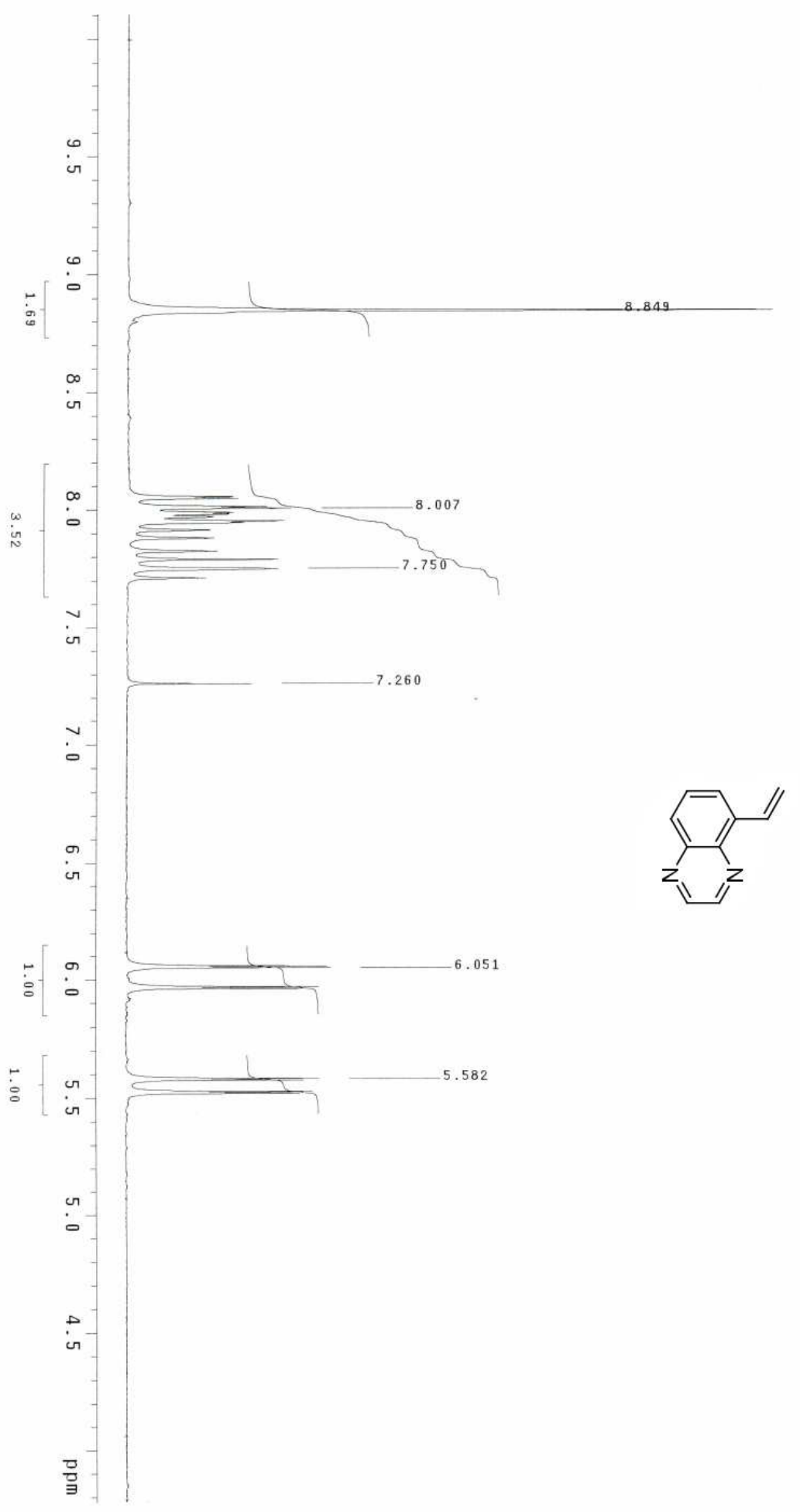


Michał Barbasiewicz et al. Structure and Activity Peculiarities of Quinoline and Quinoxaline Ruthenium Complexes...

\section{5-Vinylquinaxoline $-{ }^{1} \mathrm{H}$ NMR}
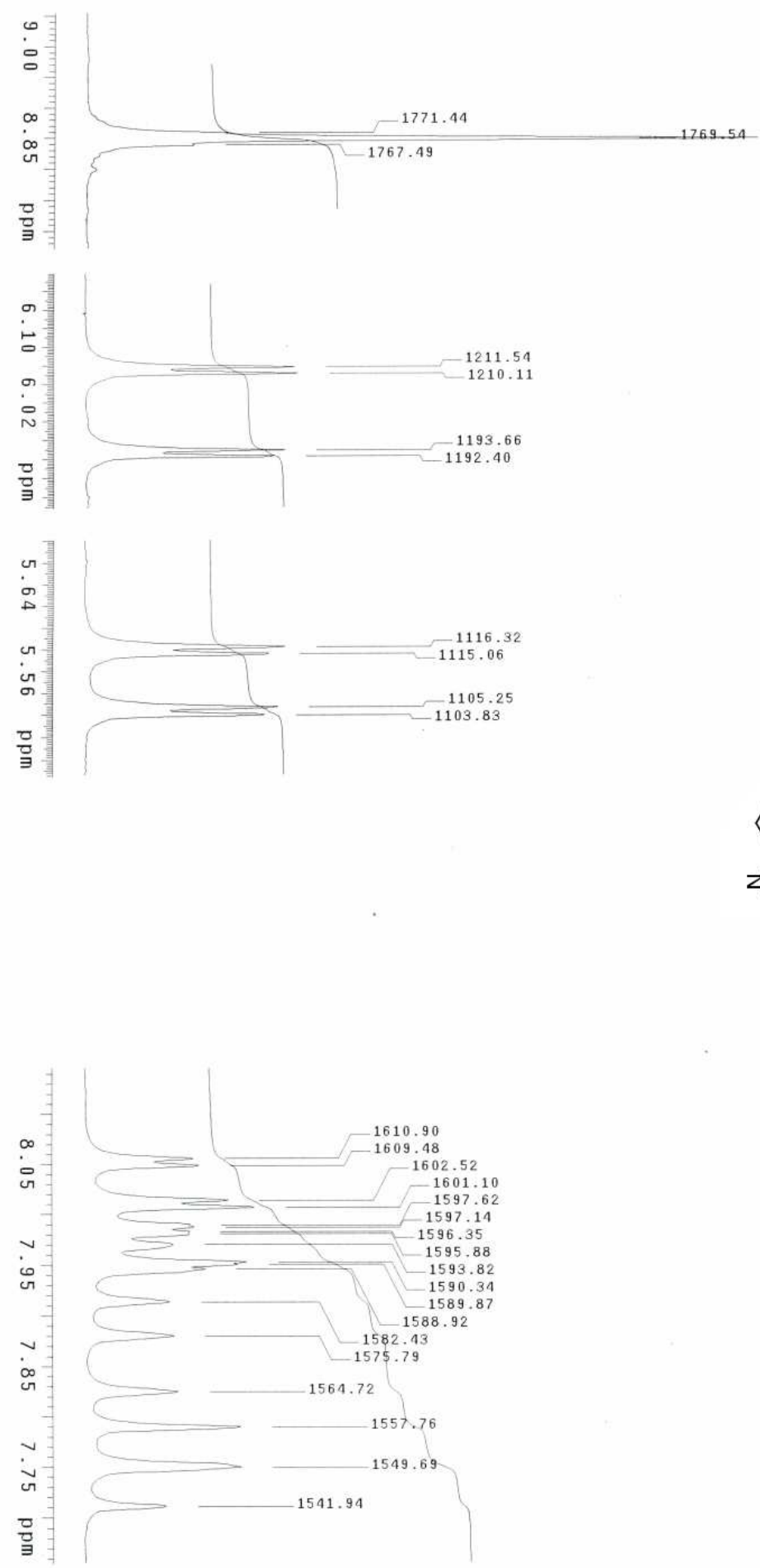
Michał Barbasiewicz et al. Structure and Activity Peculiarities of Quinoline and Quinoxaline Ruthenium Complexes...

\section{5-Vinylquinaxoline $-{ }^{13} \mathrm{C}$ NMR}
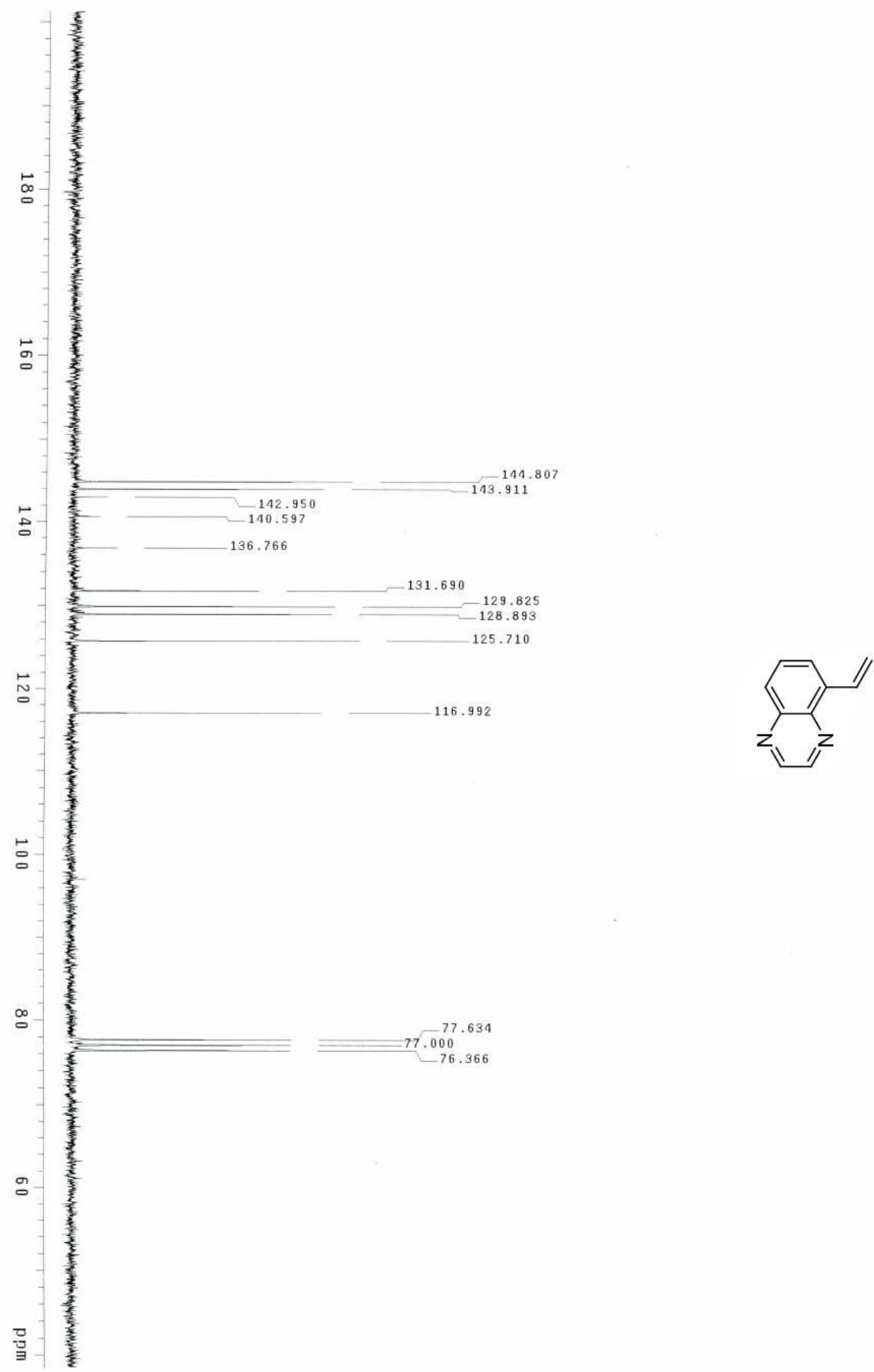
Michał Barbasiewicz et al. Structure and Activity Peculiarities of Quinoline and Quinoxaline Ruthenium Complexes. .

\section{5-Vinylquinaxoline $-{ }^{13} \mathrm{C}$ NMR}
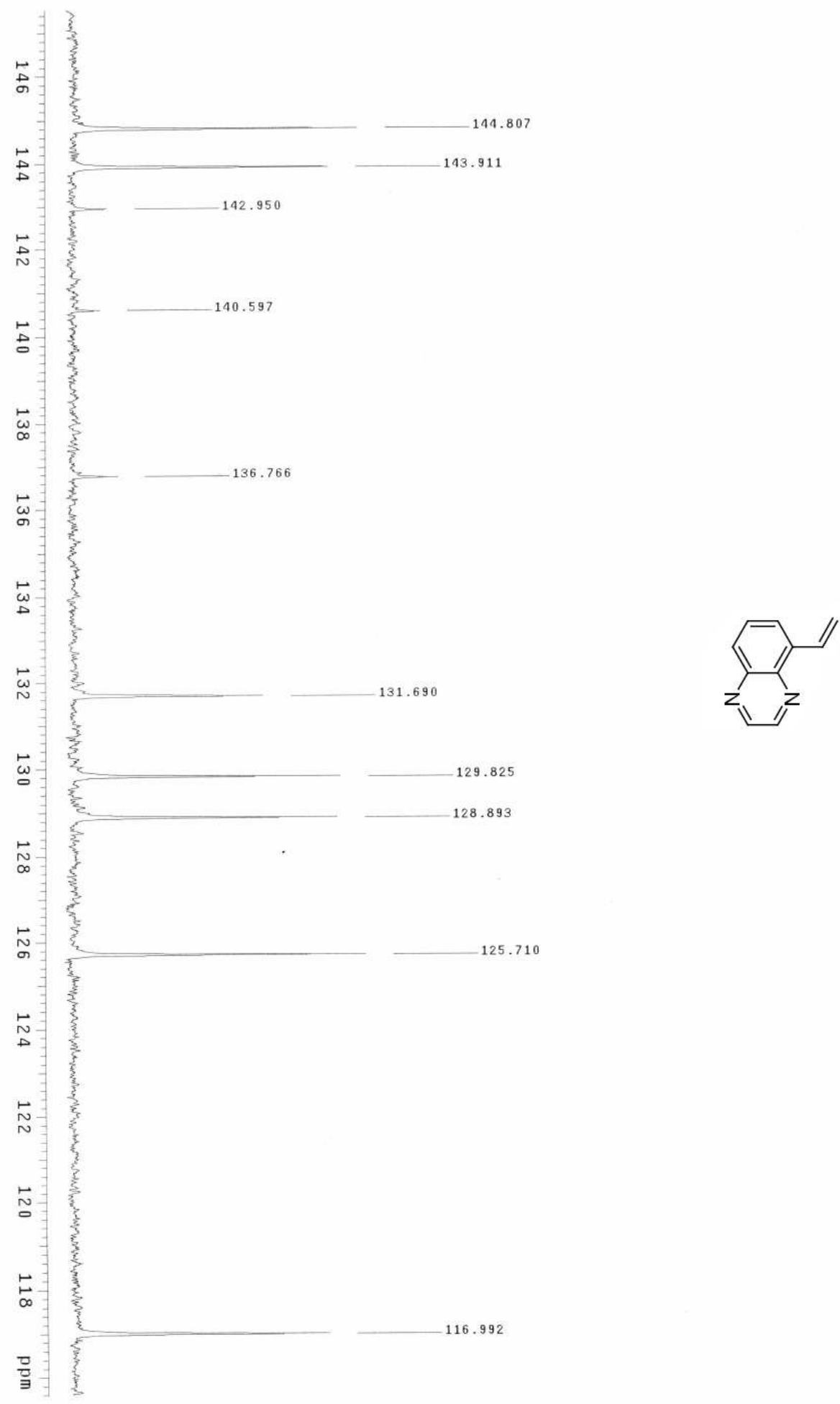


\section{Complex 1a}

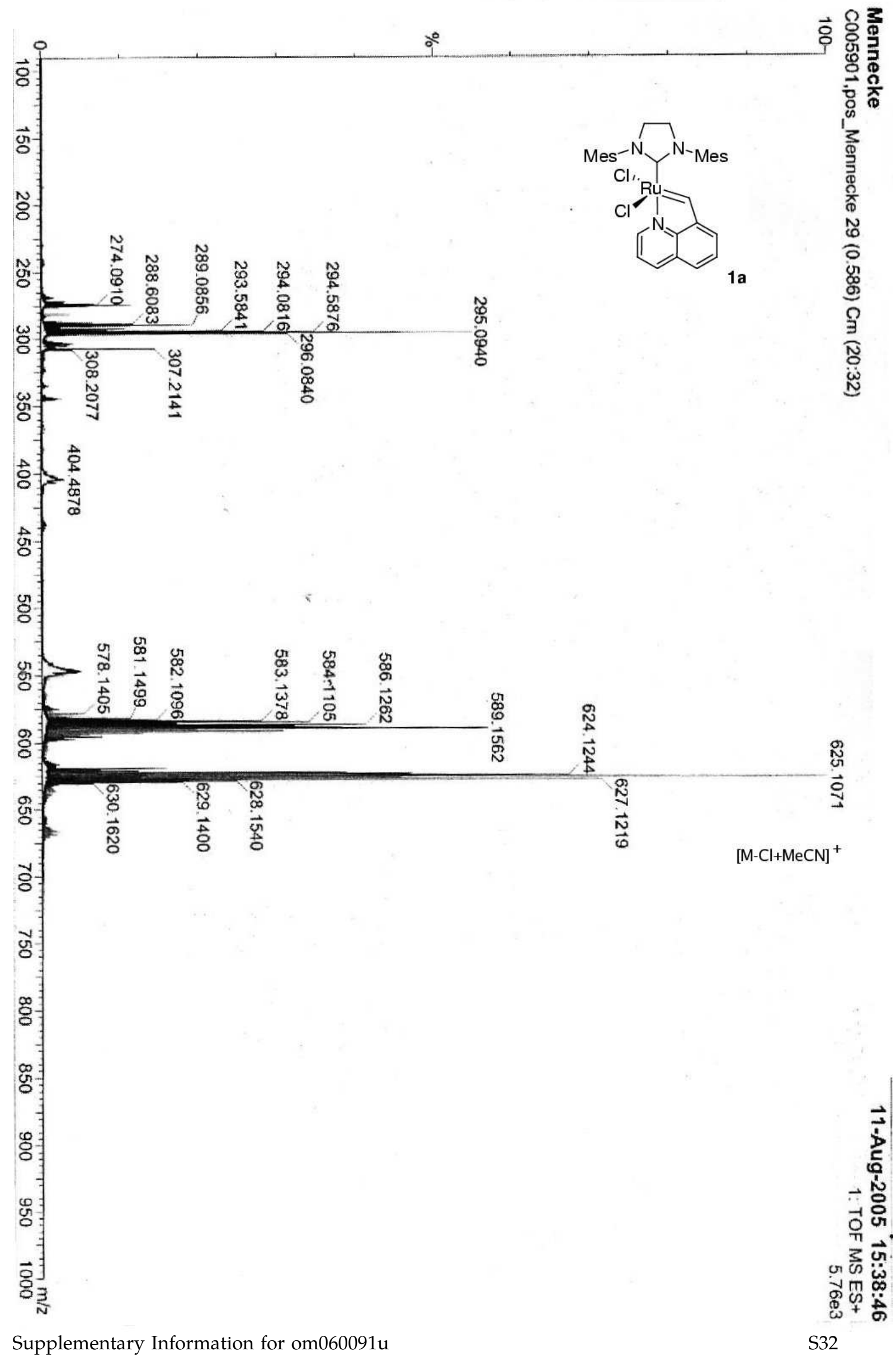


Michał Barbasiewicz et al. Structure and Activity Peculiarities of Quinoline and Quinoxaline Ruthenium Complexes...

\section{Complex 1a}

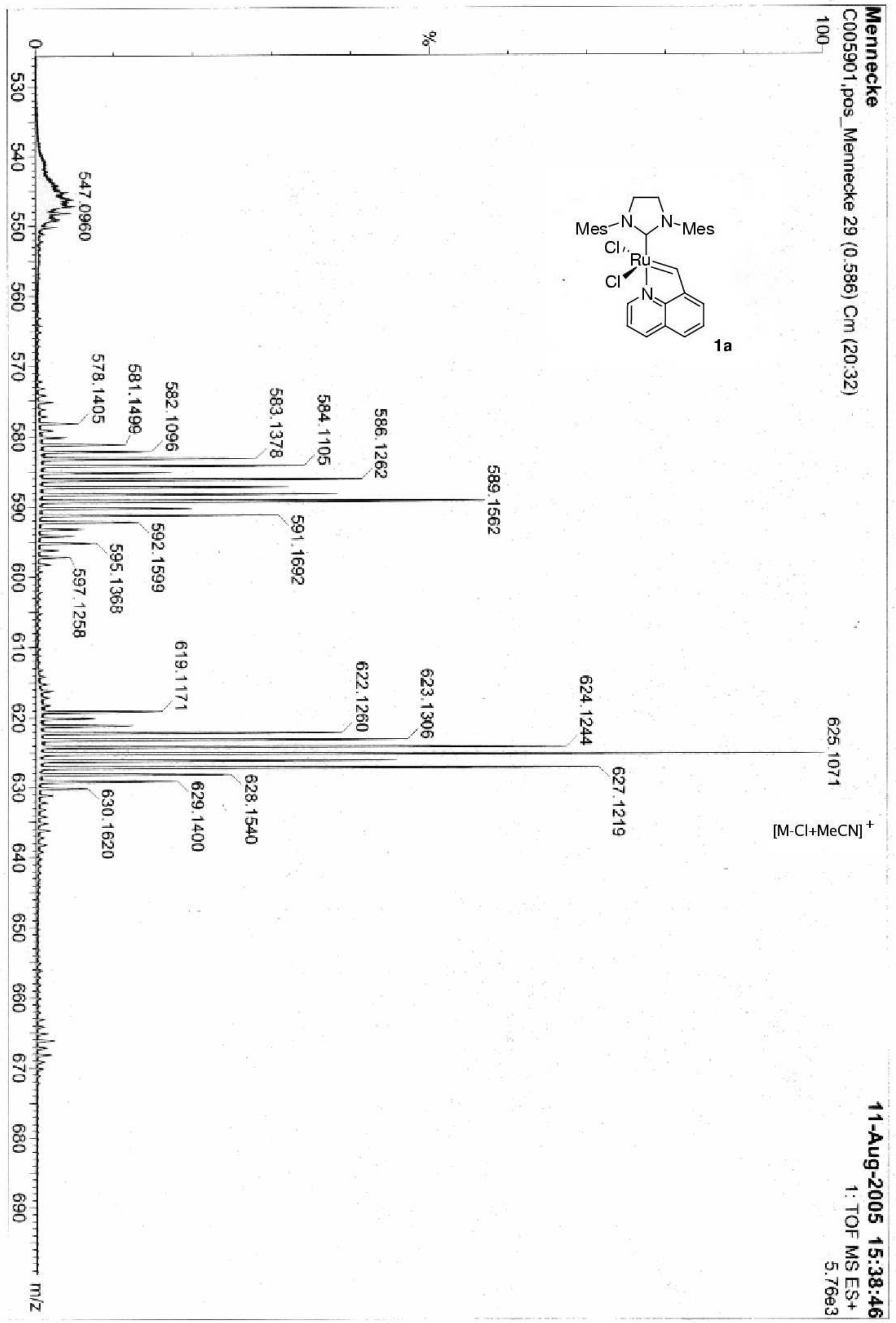




\section{Complex 2a}

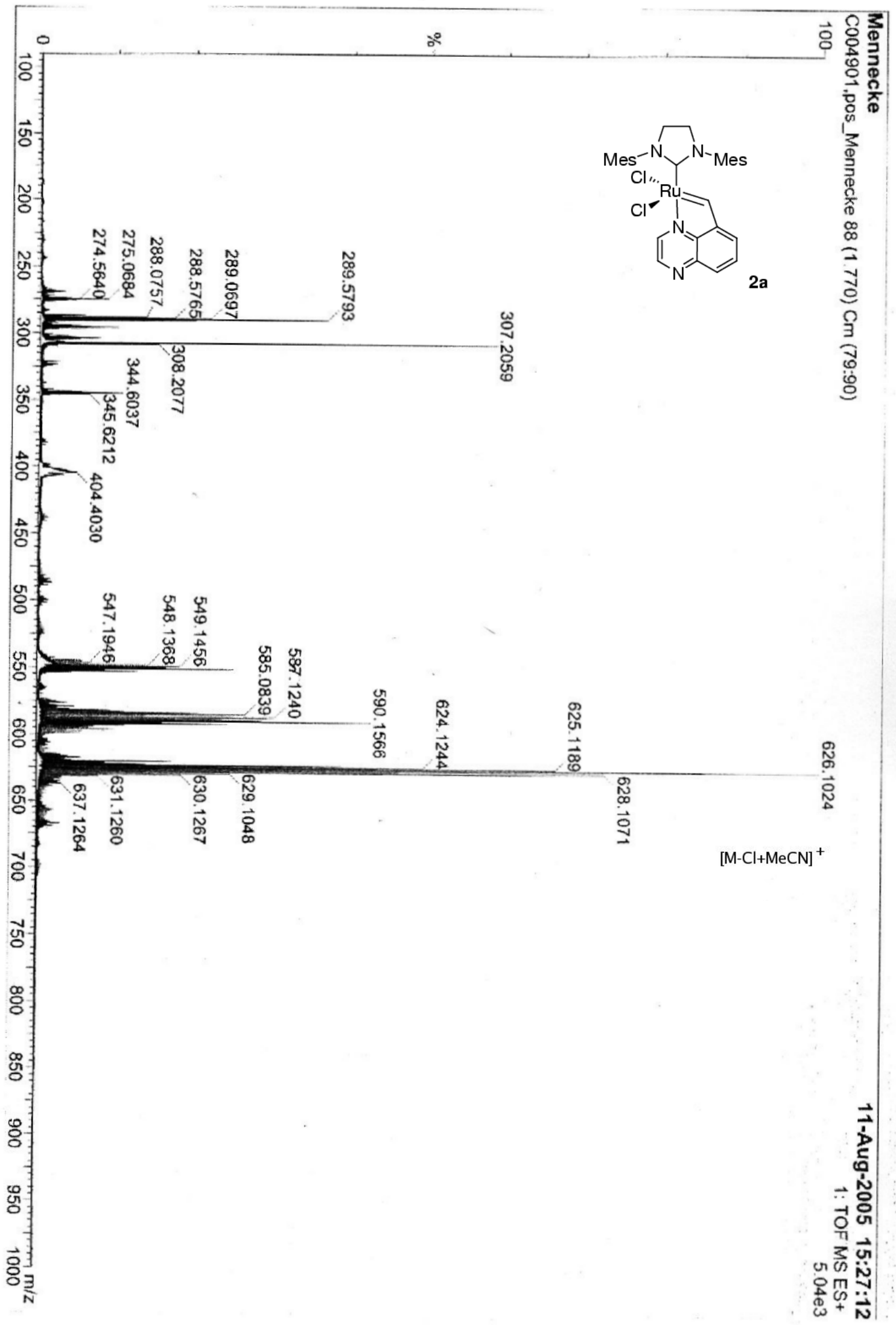




\section{Complex 2a}

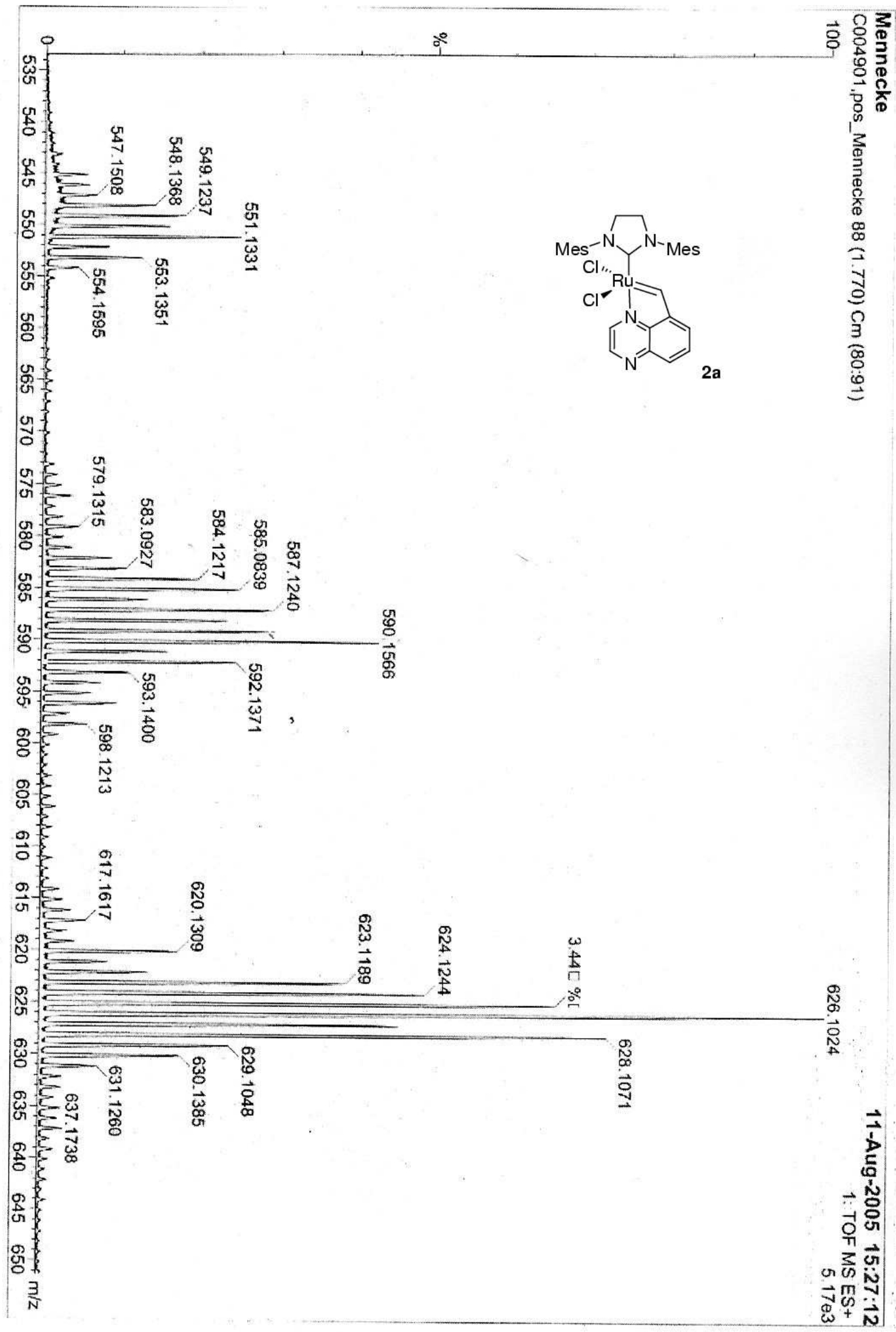


Michał Barbasiewicz et al. Structure and Activity Peculiarities of Quinoline and Quinoxaline Ruthenium Complexes...

\section{Complex 1a $-{ }^{1} \mathrm{H}$ NMR}

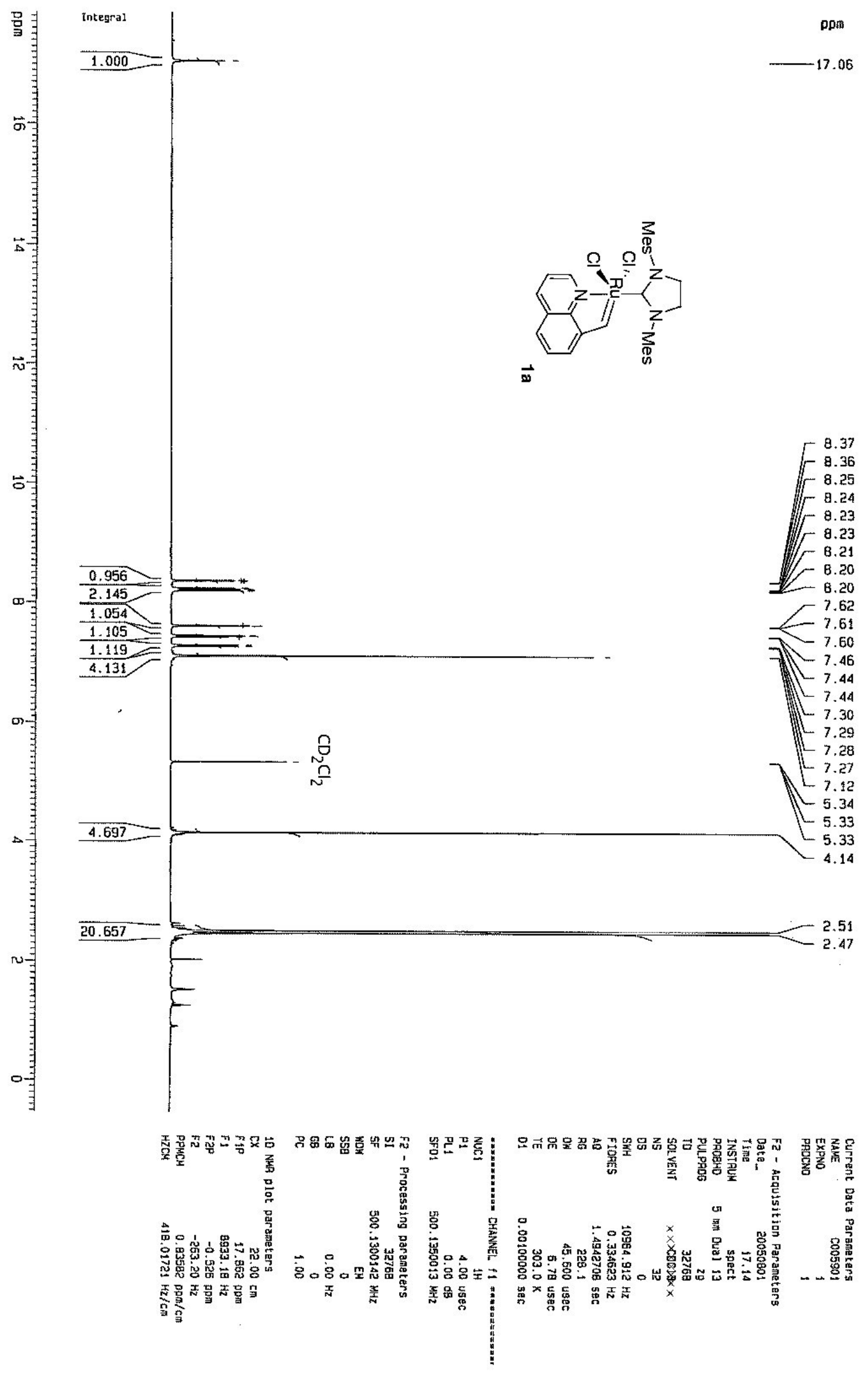


Michał Barbasiewicz et al. Structure and Activity Peculiarities of Quinoline and Quinoxaline Ruthenium Complexes...

\section{Complex 1a $-{ }^{13} \mathrm{C}$ NMR}
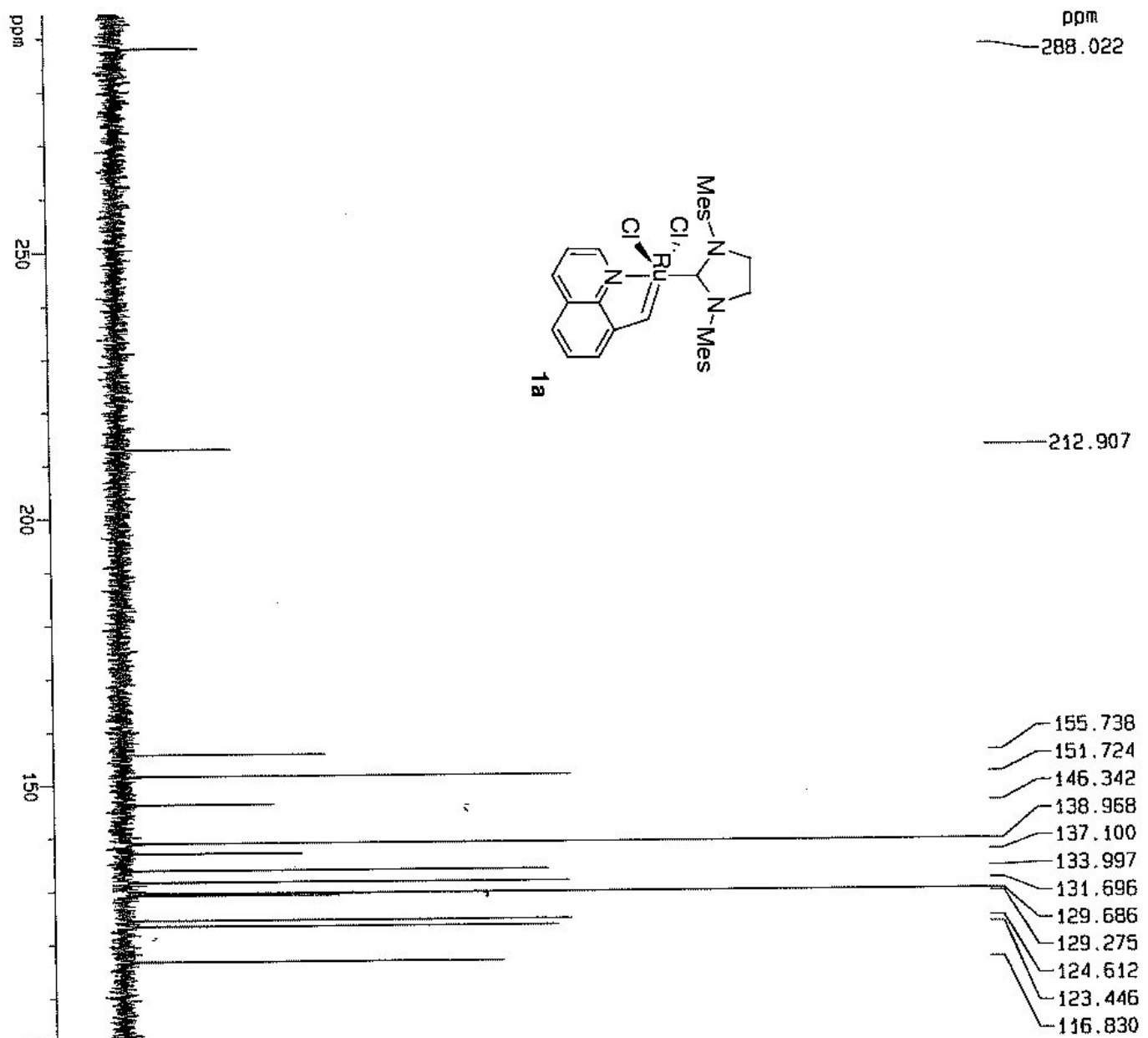

농-
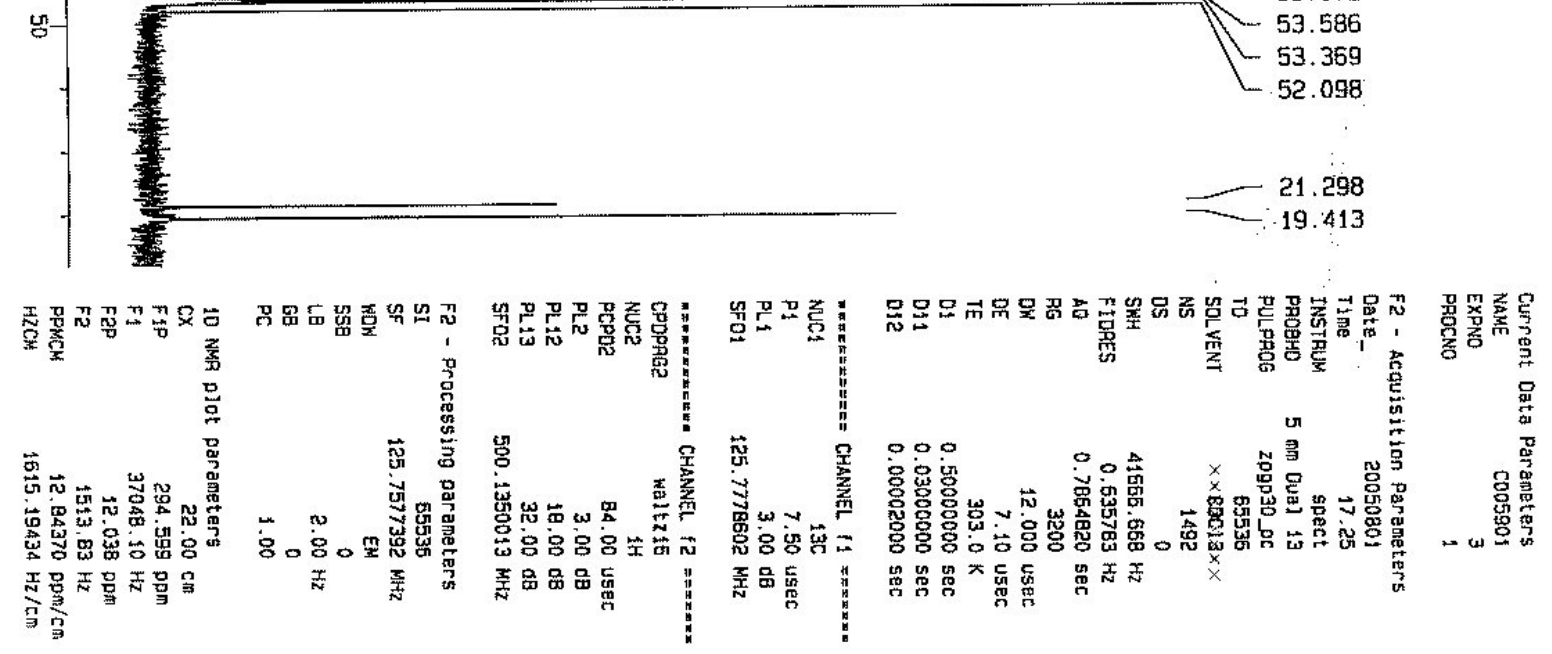
Michał Barbasiewicz et al. Structure and Activity Peculiarities of Quinoline and Quinoxaline Ruthenium Complexes...

\section{Complex $1 \mathrm{~b}-{ }^{1} \mathrm{H}$ NMR}

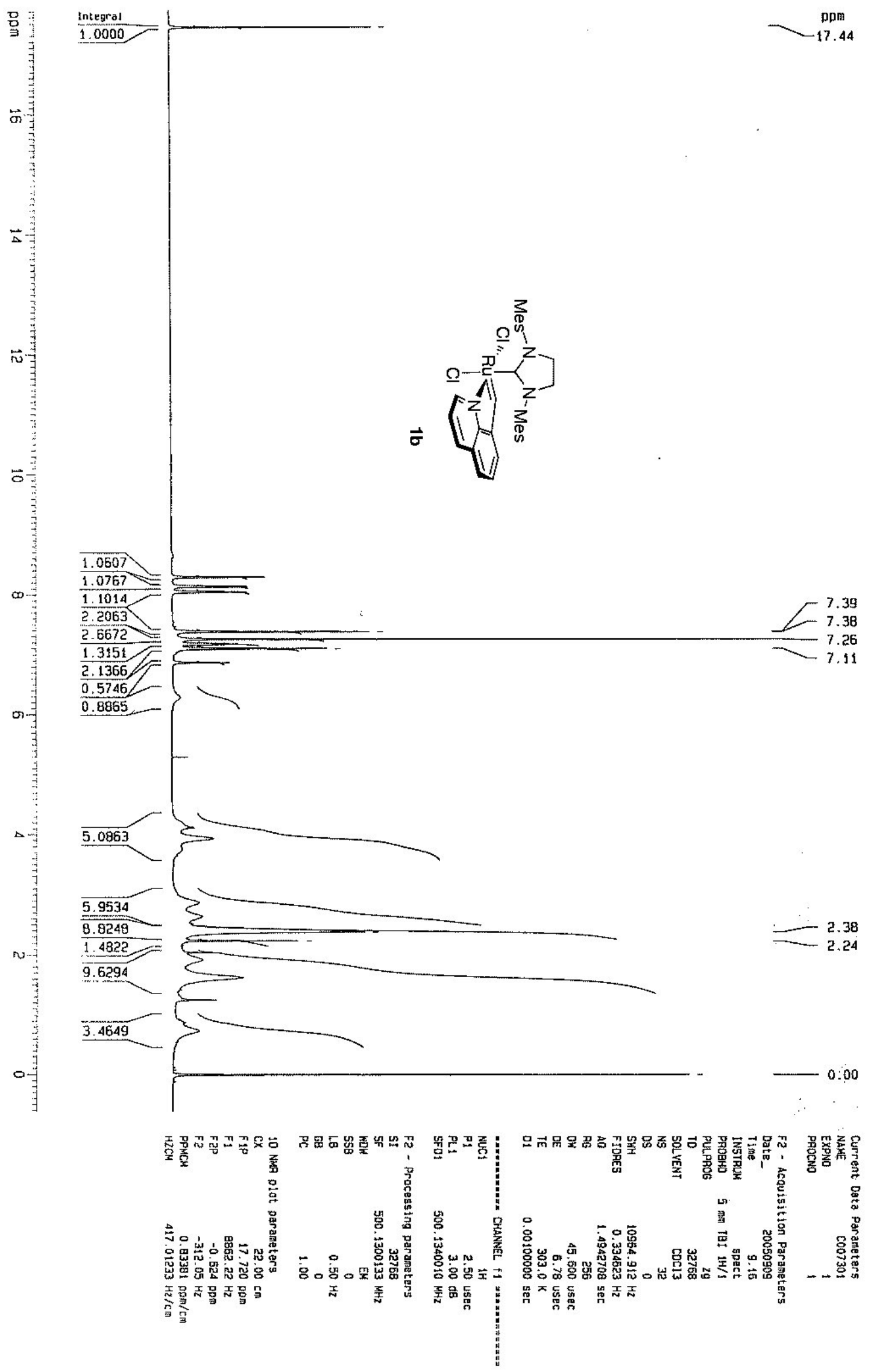


Michał Barbasiewicz et al. Structure and Activity Peculiarities of Quinoline and Quinoxaline Ruthenium Complexes...

\section{Complex $1 \mathrm{~b}-{ }^{13} \mathrm{C}$ NMR}

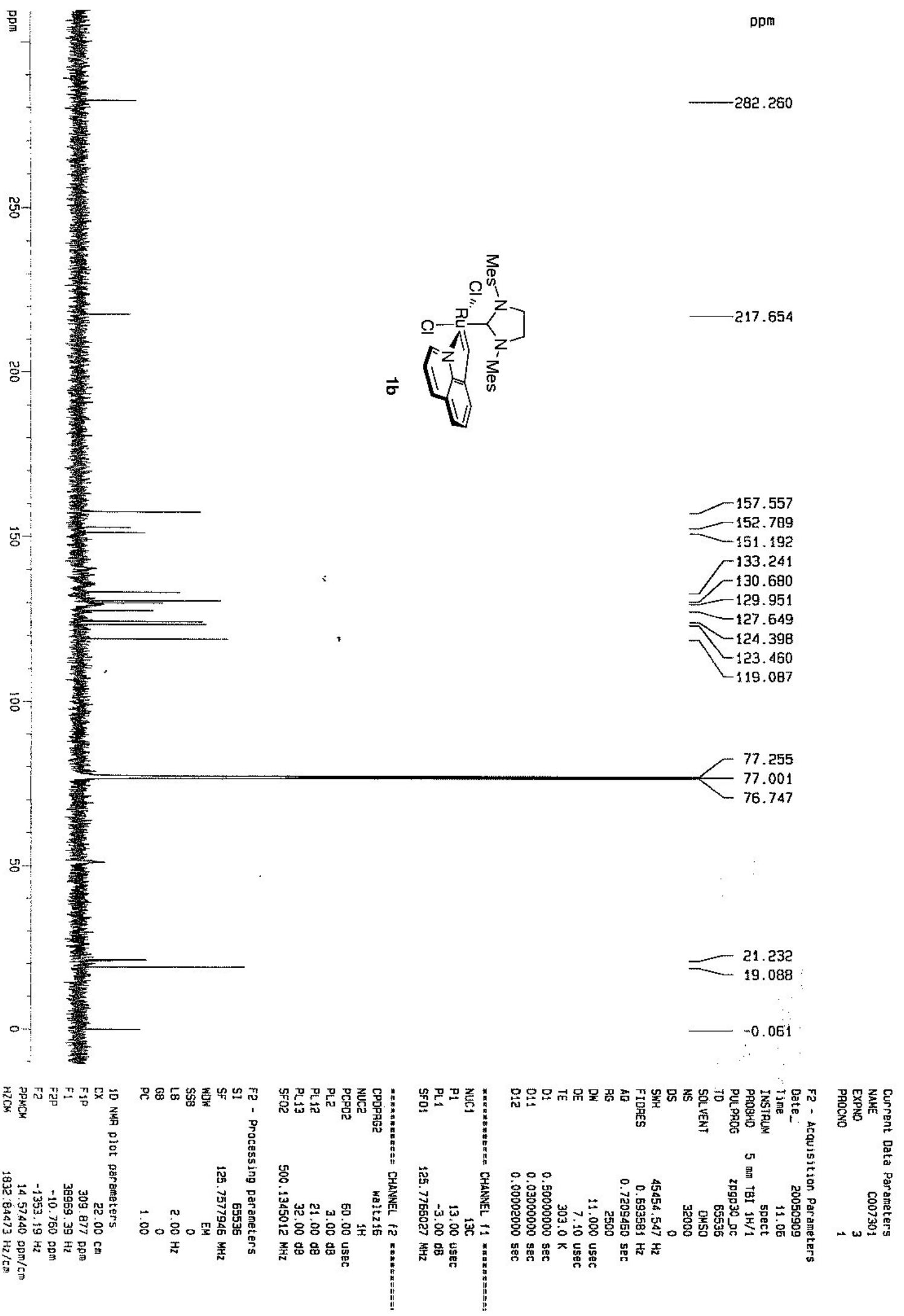




\section{Complex 2a $-{ }^{1} \mathrm{H}$ NMR}

윸

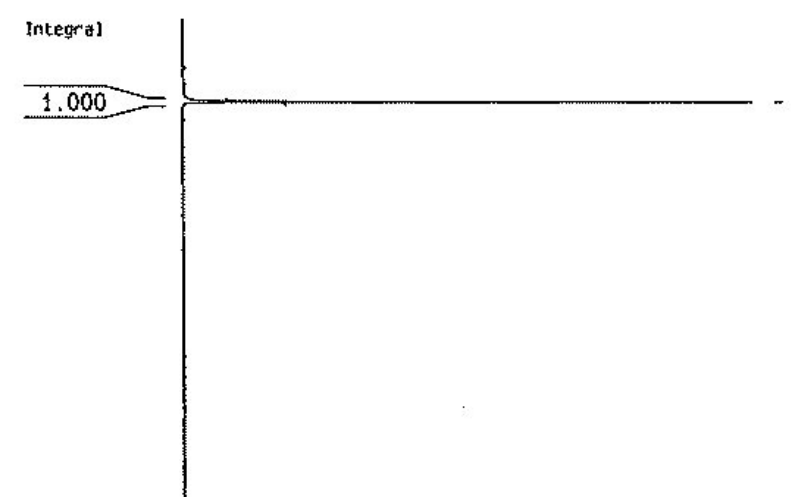

ppm

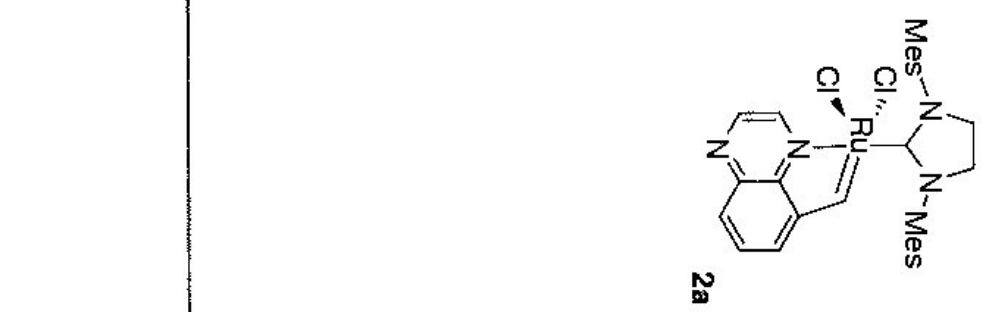

N

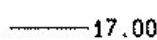

虰

$\vec{A}$

$\ddot{n}$

s-

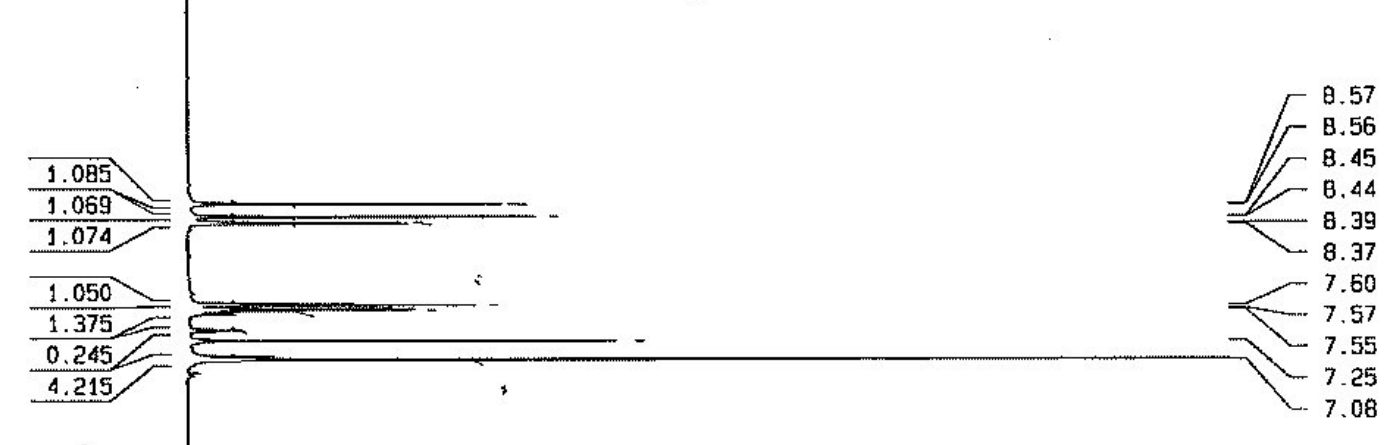

o
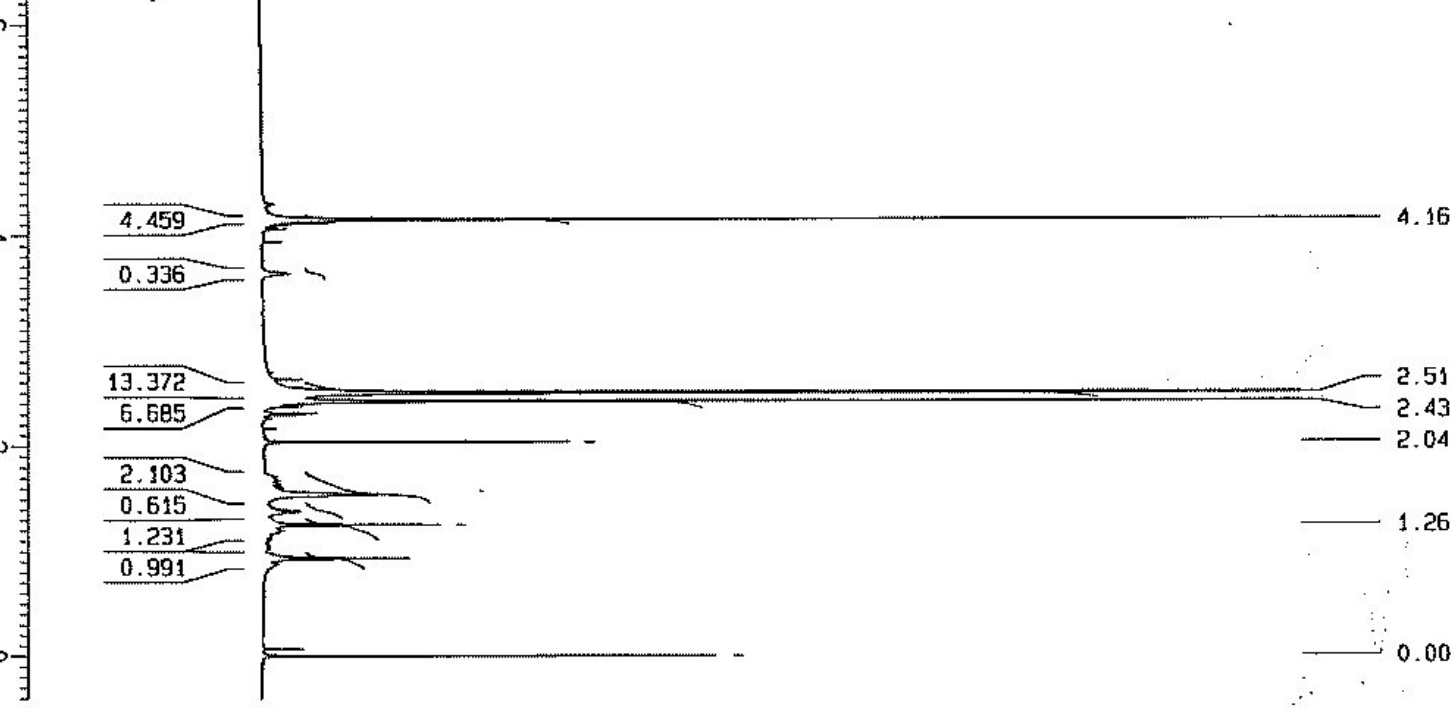

0.991

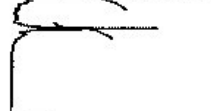

1.26

0.00

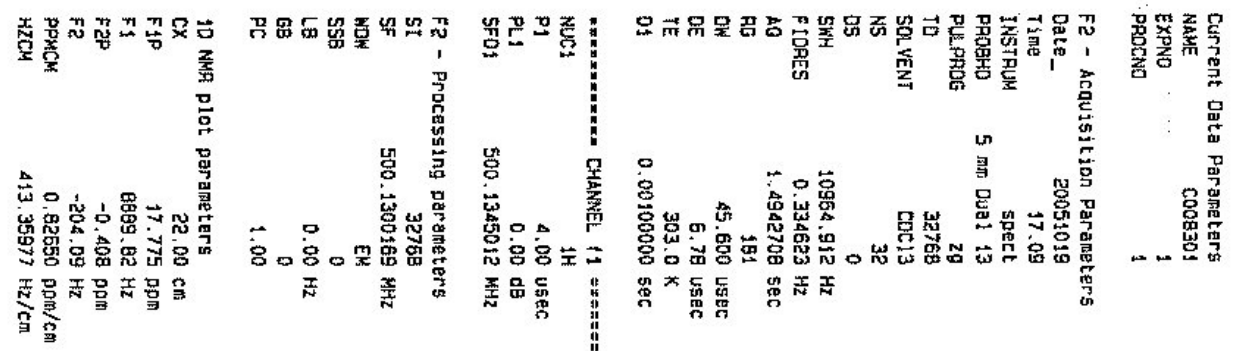




\section{Complex $2 \mathrm{a}-{ }^{13} \mathrm{C}$ NMR}

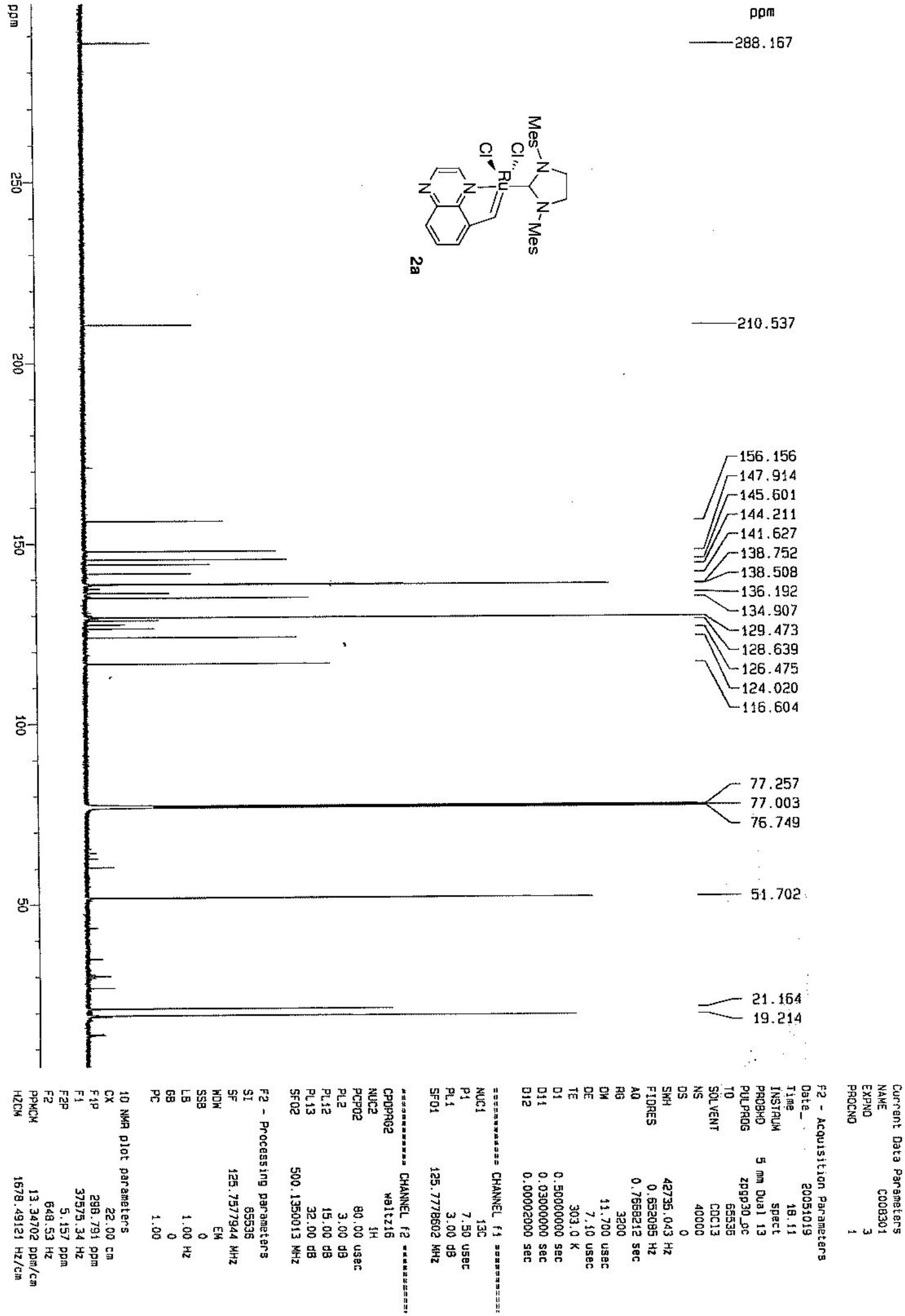


Michał Barbasiewicz et al. Structure and Activity Peculiarities of Quinoline and Quinoxaline Ruthenium Complexes...

\section{Complex $2 b-{ }^{1} \mathrm{H}$ NMR}
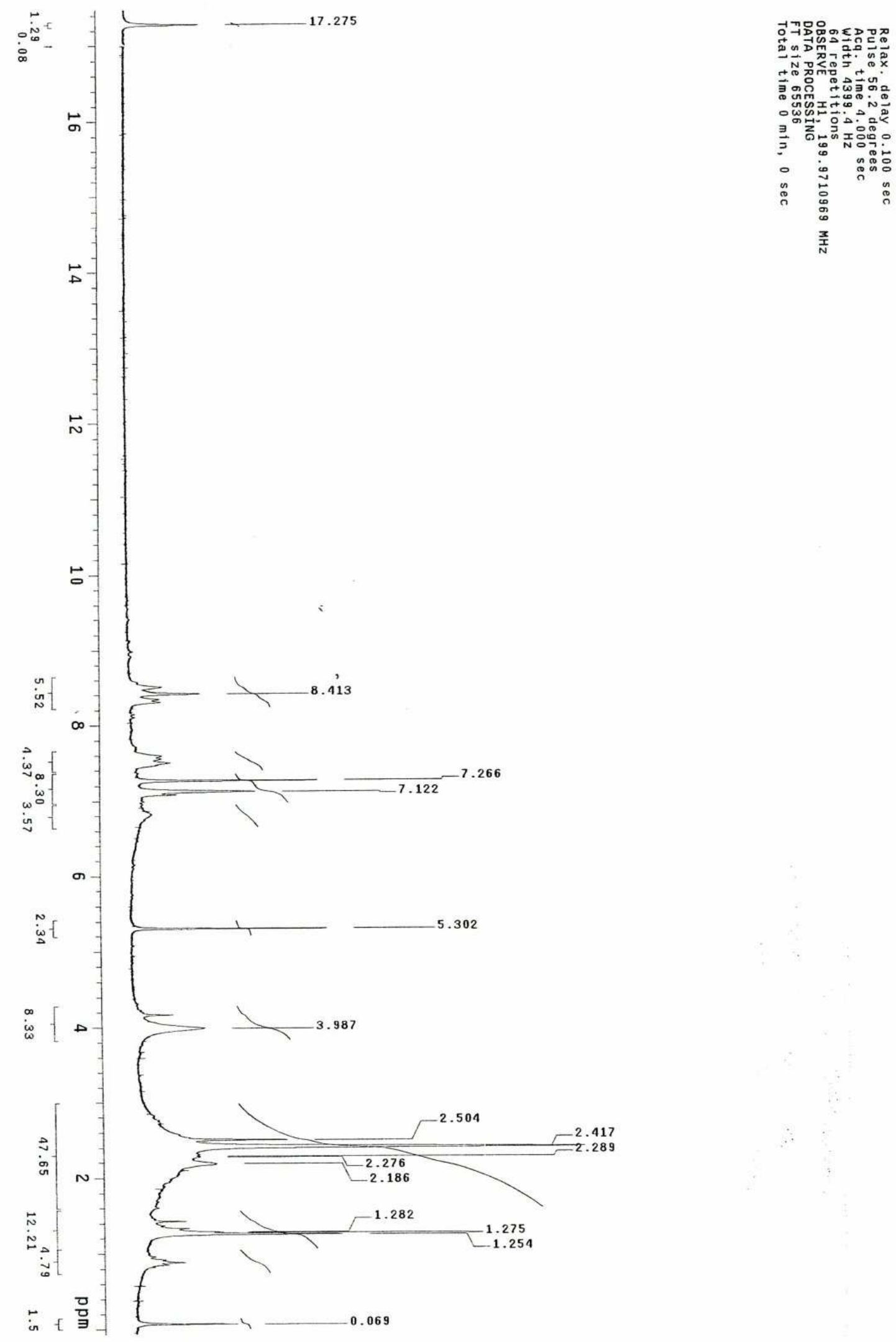